\title{
A Short Review on Polymeric Biomaterials as Additives for Lubricants
}

\author{
Gobinda Karmakar ${ }^{1}$, Koushik Dey ${ }^{1}$, Pranab Ghosh ${ }^{1}$, Brajendra K. Sharma ${ }^{2, * \mathbb{C}}$ and Sevim Z. Erhan ${ }^{3, *}$ \\ 1 Natural Product and Polymer Chemistry Laboratory, Department of Chemistry, University of North Bengal, \\ Darjeeling 734013, India; gobinda.chem@gmail.com (G.K.); koushikdey00@gmail.com (K.D.); \\ pizy12@yahoo.com (P.G.) \\ 2 Illinois Sustainable Technology Center, Prairie Research Institute, University of Illinois at Urbana-Champaign, \\ 1 Hazelwood Drive, Champaign, IL 61820, USA \\ 3 Agricultural Research Service Eastern Regional Research Center, United States Department of Agriculture, \\ Wyndmoor, PA 19038, USA \\ * Correspondence: bksharma@illinois.edu (B.K.S.); sevim.erhan@usda.gov (S.Z.E.)
}

Citation: Karmakar, G.; Dey, K.;

Ghosh, P.; Sharma, B.K.; Erhan, S.Z.

A Short Review on Polymeric

Biomaterials as Additives for

Lubricants. Polymers 2021, 13, 1333.

https://doi.org/10.3390/

polym13081333

Academic Editor: Lelia Cosimbescu

Received: 3 March 2021

Accepted: 16 April 2021

Published: 19 April 2021

Publisher's Note: MDPI stays neutral with regard to jurisdictional claims in published maps and institutional affiliations.

Copyright: (c) 2021 by the authors. Licensee MDPI, Basel, Switzerland. This article is an open access article distributed under the terms and conditions of the Creative Commons Attribution (CC BY) license (https:// creativecommons.org/licenses/by/ $4.0 /)$.

\begin{abstract}
With increasing environmental concerns and the depletion of petroleum resources, the development of lubricant additives from bioresources has attracted much attention recently. In this review, we reported a few polymers and polymer composites that are synthesized from vegetable oils (soybean oil, sunflower oil, rice bran oil, and castor oil) and used as multifunctional additives in the formulation of eco-friendly lubricant compositions. We mentioned the preparation of vegetable oilbased homo- and copolymers and their characterization by different spectral techniques (FTIR/NMR). The average molecular weights of the polymers are determined by gel permeation chromatography (GPC). Performance evaluations of the polymeric materials mainly as a viscosity index improver (VII), pour point depressant (PPD), and most importantly antifriction additives when blended with lubricating base oils are indicated. Standard ASTM methods have been applied to evaluate their performances. The findings have shown that all the additives discussed are non-toxic, biodegradable, and showed excellent performances compared to commercial petroleum-based additives.
\end{abstract}

Keywords: vegetable oils; polymerization; viscosity index; pour point; tribological performance

\section{Introduction}

The synthesis of different polymeric materials from bioresources and their application in diverse fields is a timely area of research because of several environmental impacts of commercially available nonbiodegradable polymers. Vegetable oil (VO), due to its biodegradability, low ecotoxicity, and renewability, can be used as an excellent starting material to prepare different polymeric compounds as an alternative to synthetic petroleumbased polymers in different sectors [1-4]. The application of biopolymers has been explored in different industrial sectors and products such as paints, coatings, ink resins, lubricants, and others. Good lubrication in internal combustion engines is essential to prevent energy loss, lengthen the lifetime of engines, and reduce environmental pollution. Lubricating oils are unable to meet all the requirements of modern engines, so it is essential to optimize the performance of lubricants by combining them with suitable additive packages. Additives, needed only in tiny amounts, have a very important role in improving some basic performances of lubricants such as antiwear, pour point, viscosity index, thermo-oxidative stability, etc. [5-7]. Commercially available synthetic additives prepared from fossil fuel resources (e.g., acrylate-based polymers, polycaprolactones [PCL], polyesteramides [PEA], aliphatic copolyester, ZDDP), although cheaper than biopolymers, are extremely harmful to the biosphere. They are ecotoxic and not readily biodegradable. The environmental hazards of the ZDDP additive have been well-studied in vertebrate and invertebrate species. They are considered to be toxic to aquatic wildlife with long-lasting effects. ZDDP contains 
sulfur, phosphorus, and zinc, which produce harmful emissions from engines during the tribo-chemical process [8]. An additive like ZDDP does not work well under extreme conditions of pressure and temperature. Bioadditives prepared from biooils/bioresources have the advantage over petroleum-based additives because of their eco-friendly character, multifunctional behavior, and superior tribological properties $[9,10]$.

Vegetable oil triglycerides contain long-chain fatty acids (14-22 carbons chain) with varying levels of unsaturation (Figure 1). Most vegetable oils have separate regions of nonpolar and polar groups in the same molecule. Due to the presence of polar groups, vegetable oils are amphiphilic and therefore can be used as an excellent resource to prepare both boundary and hydrodynamic lubricants [11,12]. They have many promising natural properties including good lubricity, good resistance to shear, a high flash point, a high viscosity index, and a low evaporative loss over mineral oils [13-15]. Therefore, the application of vegetable oils as feedstock to prepare bioadditives is compelling and has come into focus recently. However, because of a few shortcomings such as a high price, poor fluidity at cold temperatures, and low thermal and oxidation stability, mainly due to structural "double bond" elements in the fatty acid part and the " $\beta-\mathrm{CH}$ group" of the alcoholic components, raw vegetable oils cannot be used as a base stock/additive in lubricants [16]. This problem can be mitigated by chemically modifying vegetable oil before its application as lubricants (base stocks/additives). Among the several methods of chemical derivations [17-20], polymerization is an easy and economic synthetic technique that removes the olefinic bonds of $\mathrm{VO} /$ fatty esters and mitigates the shortcomings of their application by improving thermo-oxidative stability [20,21].

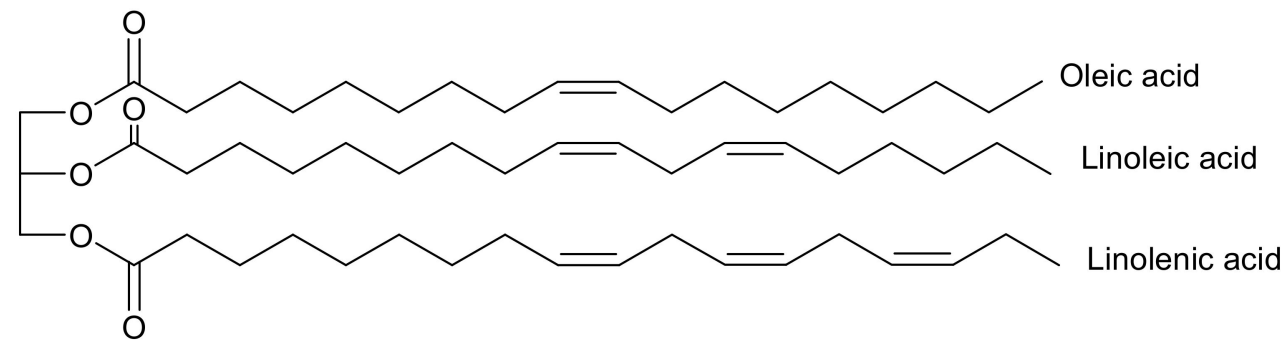

Figure 1. General structure of triglyceride esters of edible vegetable oils.

The vegetable oils/derivatives having a higher percentage of unsaturation are subjected to polymerization in different modes such as free radical, cationic, olefin metathesis, condensation polymerization, and recently, atom transfers radical polymerization (ATRP) to prepare materials with excellent additive performances [20,22]. The morphology and therefore performance of the additive are also dependent on the polymerization method used. Free radical polymerization is very effective in preparing polymeric additives that have better performances and therefore is mostly used to prepare lubricant additives [22,23]. In the polymerization, $\mathrm{VO}, \mathrm{VO}$-derived fatty acids/esters, or $\mathrm{VO}$ mixed with other materials can be used as monomers [20,22-24].

Most of the research is directed towards the synthesis of biolubricants by blending nonbiodegradable additives with chemically modified vegetable oils $[25,26]$. Yet, the formulation will be expensive since modified vegetable oils are costly compared to petroleum base stocks, and their abundance is insufficient to meet the present demand for lubricants worldwide. The blending of bioadditives from vegetable oils with mineral base stocks is currently a cost-effective and environmentally benign formulation of green lubricants. The lubricants additized with polymeric biomaterials showed an excellent tribological performance and improved the viscosity index properties and biodegradability [22-24,27-29]. The homopolymerization of functionalized triglyceride esters, free fatty acid esters of vegetable oils, or their copolymerization with suitable monomers produces biopolymers with improved additive performances and thermophysical and thermomechanical properties that have a wider range of applicability [29-31]. 
Our earlier publications mentioned the application of soybean oil, sunflower oil, castor oil, rice bran oil, palm oil, and olive oil-based polymers as multifunctional and thermally stable additives for mineral oil-based lubricants [24,27-29,32-34]. Biresaw et al. [35] discussed the application of biobased polyesters as an extreme pressure additive in mineral $(150 \mathrm{~N})$ and refined soybean base oils. The use of poly (hydroxy thioether) vegetable oil derivatives as antiwear/antifriction additives for eco-friendly lubricants has been described by Erhan et al. in U.S. Patent 7,279,448 B2 (2007) [36]. Landis [37] reported on the synthesis and evaluation of telomerized vegetable oil, sulfurized and phosphorus derivatives of telomerized vegetable oils, and combinations thereof for use as thermal-oxidative stability enhancers and viscosity improvers. Telomerization induces vegetable oil that contains no more than $4 \%$ polyunsaturated fatty acids to help enhance its thermo-oxidative stability. Recently, Nasser et al. [38] reported about the performance of the homopolymer of jojoba oil and its copolymers with different alkyl acrylates and $\alpha$-olefins as additives in lubricating oil and found amazing results.

Polymer nanocomposites have excellent wear resistance characteristics when coated on machinery parts/other materials. There are several examples of tribological performances of polymer composites. The composites of ultrahigh molecular weight polyethylene (UHMWPE) have been used as coatings to modify the surfaces of different components to protect them from wear and corrosion [39]. The composite of UHMWPE reinforced with carbon nanotube (CNT) was found as effective boundary lubricants and reduce or eliminate the usage of harmful additives in the lubricating oils. These coatings have found their way into applications ranging from microelectromechanical systems (MEMS) to demanding tribological applications such as bearings and biomedical applications [40]. The polymer composites based on vegetable oils prepared by incorporating different organic or inorganic filler particles (e.g., silica, carbon nanotubes, clay platelets, etc.) are also widely applied in different sectors such as the automotive industry [41,42], medicine [43], coating technology [44], food industry [45], preparation of printing inks [46], agrochemicals [47], aerospace [48], and more. These are used as additives to improve the tribological performances of lubricants.

In this review article (Scheme 1), we discussed in detail different vegetable oil-based polymers/polymer composites that are used as multifunctional additives for environmentally benign lubricant formulation.

\section{Vegetable Oils and Their Physicochemical Properties}

The major constituent of vegetable oils are triglyceride esters (92-98\%) of different long-chain fatty acids (saturated and unsaturated, Figure 1). The other constituents are polar lipids (phospholipids and galactolipids), monoacylglycerols, diacylglycerols, and minor amounts of free fatty acids and polyisoprenoids. Therefore, the physicochemical characteristics of vegetable oils largely depend on the fatty acid compositions in the glyceride moiety. The higher the percentage of unsaturation in the fatty acid chains, the more will be the scope of the functionalization/modification of VOs to improve their oxidative stability and cold flow properties. Therefore, VOs containing a higher percentage of unsaturation, such as soybean oil, sunflower oil, palm oil, castor oil, linseed oil, and rice bran oil, are important for preparing polymeric additives for lubricants. Among them, the nonedible vegetable oils such as neem, castor, mahua, rice bran, karanja, jatropha, and linseed, being comparatively less expensive, have an advantage over edible oils in producing additives for biofuel/biolubricants [27]. Table 1 gives a comparative look at the different fatty acid compositions with total percentages of unsaturation in different VOs. 


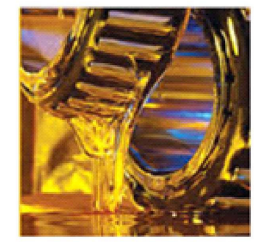

Vegetable oils (soybean/sunflower/castor/palm/rice bran/olive/almond) or their derivatives

Free radical polymerization

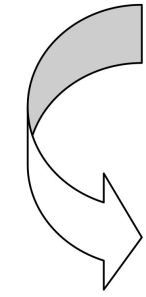

Homopolymers

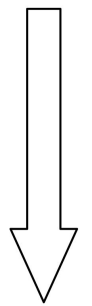

Polymer composites

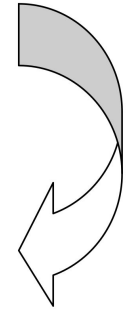

Copolymers with different monomers

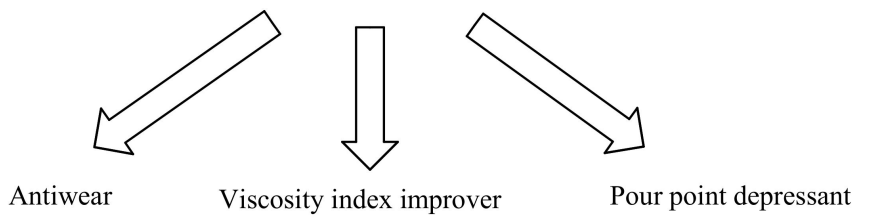

Scheme 1. Pictorial representation of the overall work.

Table 1. Fatty acid compositions of commonly used vegetable oils [20].

\begin{tabular}{cccccccccc}
\hline Vegetable Oils & C12:0 & C14:0 & C16:0 & C18:0 & C16:1 & C18:1 & C18:2 & C18:3 & Others \\
\hline Soybean oil & - & - & $11-12$ & 3 & 0.2 & 24 & $53-55$ & $6-7$ & - \\
Sunflower oil & - & - & 7 & 5 & 0.3 & $20-25$ & $63-68$ & 0.2 & - \\
Rapeseed oil & - & - & $4-5$ & $1-2$ & 0.21 & $56-64$ & $20-26$ & $8-10$ & $9.1(20: 1)$ \\
Palm oil & - & 1 & $37-41$ & $3-6$ & 0.4 & $40-45$ & $8-10$ & - & - \\
Rice bran oil & - & - & $20-22$ & $2-3$ & 0.19 & 42 & 31 & 1.1 & - \\
Cottonseed oil & - & 1 & $22-26$ & $2-5$ & 1.4 & $15-20$ & $49-58$ & - & - \\
Coconut oil & $44-52$ & $13-19$ & $8-11$ & $1-3$ & - & $5-8$ & $0-1$ & - & - \\
Corn (Maize) oil & - & - & $11-13$ & $2-3$ & 0.3 & $25-31$ & $54-60$ & 1 & - \\
Peanut/Groundnut & - & - & $10-11$ & $2-3$ & 0 & $48-50$ & $39-40$ & - & - \\
Sesame oil & - & - & $7-11$ & $4-6$ & 0.11 & $40-50$ & $35-45$ & - & - \\
Safflower oil & - & - & $5-7$ & $1-4$ & 0.08 & $13-21$ & $73-79$ & - & - \\
Karanja oil & - & - & $11-12$ & $7-9$ & - & 52 & $16-18$ & - & - \\
Jatropha oil & - & 1.4 & $13-16$ & $6-8$ & - & $38-45$ & $32-38$ & - & - \\
Rubber seed oil & - & $2-3$ & 10 & 9 & - & 25 & 40 & 16 & - \\
Mahua oil & - & - & 28 & 23 & - & $41-51$ & $10-14$ & - \\
Tung oil & - & - & 2.67 & 2.4 & - & 7.88 & 6.6 & $80.46^{*}$ & - \\
Neem oil & - & - & 18 & 18 & - & 45 & $18-20$ & 0.5 & - \\
Castor oil & - & - & $0.5-1$ & $0.5-1$ & - & $4-5$ & $2-4$ & $0.5-1$ & $83-85^{\#}$ \\
Linseed oil & - & - & $4-5$ & $2-4$ & $0-0.5$ & 19.1 & $12-18$ & 56.6 & - \\
Olive oil & - & - & 13.7 & 2.5 & 1.8 & 71 & 10 & $0-1.5$ & - \\
\hline
\end{tabular}

* Alpha-eleostearic acid. " Ricinoleic acid.

The quality and performance of an additive depend largely on the physical properties of the $\mathrm{VO}$ from which it was derived. A vegetable oil having a higher viscosity index, flash point, thermo-oxidation stability, shear stability, and lower pour point and cloud point produce additives with enhanced performances [27]. The physical characteristics of different VOs are listed in Table 2. 
Table 2. Physical properties of vegetable oils [20].

\begin{tabular}{|c|c|c|c|c|c|c|}
\hline Vegetable Oils & $\begin{array}{l}\text { Iodine } \\
\text { Value }\end{array}$ & $\begin{array}{l}\text { Pour Point } \\
\left({ }^{\circ} \mathrm{C}\right)\end{array}$ & $\begin{array}{l}\text { Cloud Point } \\
\left({ }^{\circ} \mathrm{C}\right)\end{array}$ & $\begin{array}{l}\text { Kinematic Viscosity } \\
\text { at } 40{ }^{\circ} \mathrm{C}\left(\mathrm{mm}^{2} / \mathrm{s}\right)\end{array}$ & $\begin{array}{c}\text { Flash Point } \\
\left({ }^{\circ} \mathrm{C}\right)\end{array}$ & $\begin{array}{c}\text { Density at } \\
15^{\circ} \mathrm{C}\left(\mathrm{g} / \mathrm{cm}^{3}\right)\end{array}$ \\
\hline Soybean oil & $138-143$ & -12 & -4 & 29 & 254 & 0.914 \\
\hline Sunflower oil & $125-140$ & -15 & -9.5 & 36 & 274 & 0.916 \\
\hline Rapeseed oil & 98-105 & -15 & -2 & 35 & 246 & 0.912 \\
\hline Palm oil & $48-58$ & 23.6 & 25.2 & 39.4 & 252 & 0.919 \\
\hline Rice bran oil & 103 & 13 & 16 & 38.2 & 184 & 0.906 \\
\hline Cotton seed oil & 90-119 & -4.5 & -0.5 & 34 & 234 & 0.918 \\
\hline Coconut oil & $8-11$ & 12.7 & 13.1 & 27 & 266 & 0.918 \\
\hline $\begin{array}{l}\text { Peanut/Ground } \\
\text { nut/Arachis oil }\end{array}$ & $84-100$ & -7 & 4.5 & 40 & 271 & 0.903 \\
\hline Sesame oil & $104-116$ & -11 & -8 & 36 & 260 & 0.918 \\
\hline Karanja oil & $81-90$ & -4 & 2 & 38.8 & 212 & 0.9358 \\
\hline Jatropha oil & 82-98 & -6 & 11 & 34 & 225 & 0.94 \\
\hline Rubber seed oil & 104 & 18 & 25 & 33.89 & 228 & 0.928 \\
\hline Mahua oil & $58-70$ & 11 & 20 & 37.18 & 238 & 0.945 \\
\hline Neem oil & 81 & 7 & 13 & 35.8 & 200 & 0.918 \\
\hline Castor oil & $83-86$ & -21 & -18 & 251 & 229 & 0.960 \\
\hline Linseed oil & $168-204$ & -15 & 5 & $26-29$ & 241 & 0.938 \\
\hline Safflower oil & 145 & -7 & -2 & 28.3 & 260 & 0.914 \\
\hline Olive oil & $75-94$ & -14 & -11 & 39 & 177 & 0.918 \\
\hline
\end{tabular}

3. Polymerization of Different Vegetable Oils/Derivatives, Their Characterization, and Application as Lubricant Additives

Polymerization is a unique technique through which the reactive olefinic bonds of $\mathrm{VO} /$ derivatives are involved, and therefore the oxidative stability and cold flow properties of the oils are improved. The homopolymerization of $\mathrm{VO} /$ derivatives or their copolymerization with suitable monomers can be performed in different ways, as mentioned above. Here, we have discussed the polymers/composites synthesized via the free radical polymerization pathway. Homopolymers of vegetable oil and their copolymers with different functional molecules (alkyl acrylates, long-chain alkenes, styrene) were prepared by thermal/microwave methods using radical initiators like azobisisobutyronitrile (AIBN), benzoyl peroxide (BZP) $[24,28]$. The characterizations were performed by different spectral (FTIR/NMR) techniques. The average molecular weights were determined by GPC. The characterization data (FTIR, NMR, GPC) of some polymers are mentioned in Table 3. The individual descriptions of the different vegetable oil-based polymers as lubricant additives are depicted below.

The IR and ${ }^{1} \mathrm{HNMR}$ absorption peaks in the range $1732-1745 \mathrm{~cm}^{-1}$ and $4.031-4.9 \mathrm{ppm}$, respectively in Table 3 indicated the presence of ester carbonyl groups of the polymers. The C-O stretching vibrations of ester carbonyl groups of all the polymers appeared within the range 1051-1270 $\mathrm{cm}^{-1}$. The presence of the methylene and methyl groups of the fatty acid chains is also proved by the respective IR and ${ }^{1} \mathrm{HNMR}$ absorption peaks in Table 3 . The GPC data in the above table gives some idea about the average molecular weights of the biobased polymers. The values of the average molecular weight depend on the degree of polymerization, cross-linking of the monomers/unsaturated fatty esters, and the average size of polymer molecules. These further depend upon several factors such as the degree of unsaturation of different vegetable oils, the type of monomers (in case of copolymerization), and the methods and conditions applied on polymerization (time, ratio of monomers, initiators, temperature, etc.). The homopolymer of vegetable oils like SBO, SFO containing a higher percentage of unsaturation showed comparatively higher average molecular weights. When acrylate chains are introduced in the backbone of triglyceride esters of vegetable oils, the average molecular weights are increased due to a higher degree of polymerization which is reflected on their VI values, also mentioned later. Terpolymers have a comparatively low value of the average molecular weight, probably due to a shorter chain length of each polymer unit. 
Table 3. Characterization data of different vegetable oil-based polymers.

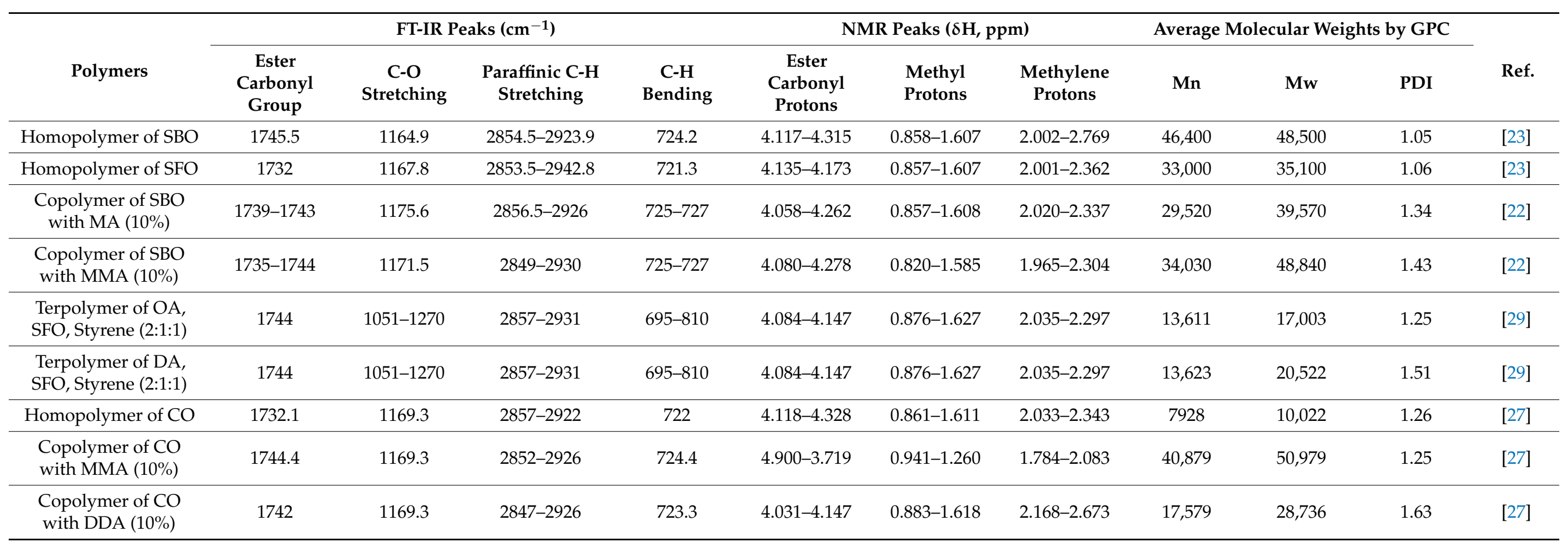




\subsection{Polymers of Soybean Oil/Derivatives}

After palm oil, soybean oil (SBO) is the second most abundant vegetable oil with a production of around 50 million metric tons of the 209.14 million metric tons of all vegetable oils produced in 2020-2021, according to statistical reports. The United States occupied the second position in the production of soybean oil after China. The unsaturation in SBO is around $85 \%$, which makes it suitable for producing different polymeric additives. In our previous work, the homopolymer of soybean oil and its copolymers with different monomers, such as methyl acrylate (MA), 1-decene, and styrene, at different percentage levels have been synthesized by a thermal method using azobisisobutyronitrile (AIBN) as a radical initiator [24]. All the polymers showed excellent multifunctional performances as additives in lubricants. The viscosity index (VI), antiwear, and pour point (PP) properties of the base fluids were enhanced significantly by the addition of these additives. Polymerization increased the thermo-oxidative stability of soybean oil also. Table 4 shows the VI and PP values of lubricant compositions blended with additives at different percentages.

Table 4. Viscosity index and pour point values of lubricants with additives at different concentrations [24].

\begin{tabular}{|c|c|c|c|c|c|c|c|c|c|c|c|}
\hline \multirow{2}{*}{ Sample } & \multirow{2}{*}{ Base Oil } & \multicolumn{5}{|c|}{ VI } & \multicolumn{5}{|c|}{ Pour Point $\left(-{ }^{\circ} \mathrm{C}\right)$} \\
\hline & & $0 \%$ & $2 \%$ & $3 \%$ & $4 \%$ & $5 \%$ & $0 \%$ & $2 \%$ & $3 \%$ & $4 \%$ & $5 \%$ \\
\hline \multirow{2}{*}{ S-1 } & SN1 & 80 & 132 & 170 & 211 & 227 & -3 & -3 & -7 & -8 & -12 \\
\hline & SN2 & 89 & 140 & 200 & 227 & 256 & -6 & -6 & -8 & -9 & -15 \\
\hline \multirow{2}{*}{$\mathrm{S}-2$} & SN1 & 80 & 113 & 127 & 135 & 138 & -3 & -6 & -9 & -12 & -18 \\
\hline & SN2 & 89 & 113 & 133 & 153 & 177 & -6 & -8 & -12 & -15 & -20 \\
\hline \multirow{2}{*}{ S-3 } & SN1 & 80 & 116 & 134 & 145 & 160 & -3 & -6 & -8 & -9 & -15 \\
\hline & SN2 & 89 & 122 & 144 & 173 & 189 & -6 & -8 & -10 & -12 & -15 \\
\hline \multirow{2}{*}{ S-4 } & SN1 & 80 & 156 & 199 & 232 & 240 & -3 & -3 & -7 & -9 & -9 \\
\hline & SN2 & 89 & 150 & 212 & 234 & 262 & -6 & -6 & -8 & -9 & -10 \\
\hline \multirow{2}{*}{ S-5 } & SN1 & 80 & 142 & 184 & 218 & 232 & -3 & -3 & -7 & -9 & -9 \\
\hline & SN2 & 89 & 152 & 210 & 242 & 270 & -6 & -6 & -6 & -8 & -10 \\
\hline \multirow{2}{*}{ S-6 } & SN1 & 80 & 162 & 201 & 232 & 242 & -3 & -3 & -6 & -9 & -12 \\
\hline & SN2 & 89 & 166 & 211 & 244 & 272 & -6 & -6 & -8 & -10 & -15 \\
\hline \multirow{2}{*}{ S-7 } & SN1 & 80 & 152 & 192 & 217 & 236 & -3 & -5 & -7 & -8 & -12 \\
\hline & SN2 & 89 & 162 & 199 & 231 & 268 & -6 & -6 & -8 & -9 & -15 \\
\hline
\end{tabular}

S-1: homopolymer of SBO; S-2: SBO + 10\% MA; S-3: SBO + 5\% MA; S-4: SBO + 10\% 1-Decene; S-5: SBO + 5\% 1-Decene; S-6: SBO + 10\% Styrene; S-7: SBO + 5\% Styrene.

The data indicated that the VI values of the base oils blended with additives are higher compared to those without additives. The results also showed that the VI values gradually increase with an increase in the additive concentration of the base fluids. The homopolymer of SBO showed excellent results. The incorporation of styrene and 1-decene to SBO enhanced the VI values, whereas copolymers of SBO with MA (S-2 and S-3) showed the least VI value. Moreover, it was found that all the polymers have much higher VI values compared to commercially available acrylate-based or olefin polymers. The pour point value of the copolymer of soybean oil with MA exhibited a better pour point depressant (PPD) performance compared to the others. The soy-acrylate copolymers, due to their more polar nature, fight better against the formation of wax crystals and therefore show higher pour point values that are comparable with those of commercially available polymethacrylates.

The tribological properties in terms of wear scar diameter (WSD) and COF (coefficient of friction) of the lubricant compositions prepared by blending the polymers at different 
concentrations in SN1 oil were determined by a four-ball wear test apparatus (FBWT) at $392 \mathrm{~N}$ applied load. Figures 2 and 3 showed the WSD and COF values of the lubricants with different percentages of additives. As indicated by the figures, the copolymers of SBO with MA and 1-decene perform better as antiwear additives. With increasing additive concentrations, both the WSD and COF decrease. S-2 showed the highest reduction of the WSD in the SN1 base oil at a 0.05 mass fraction $(41.8 \%)$, whereas the homopolymer of SBO was minimal $(26.8 \%)$.

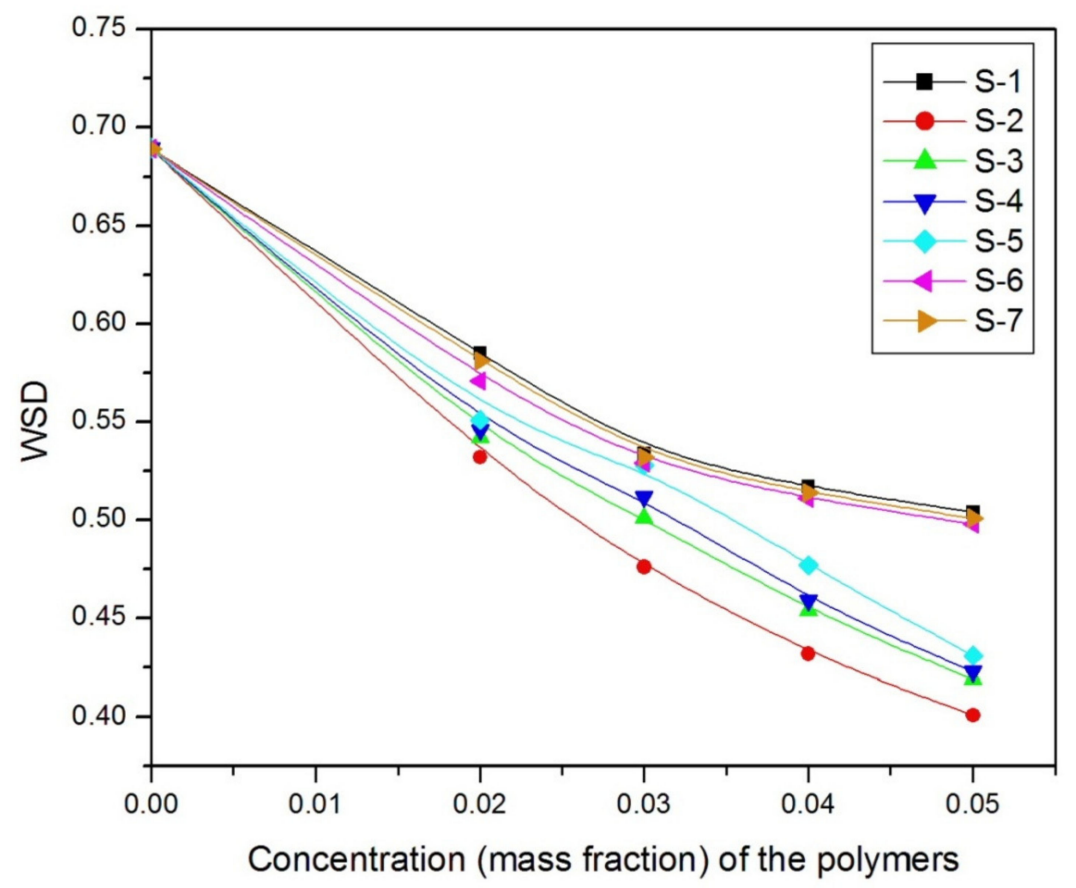

Figure 2. Wear scar diameter (WSD) in mm of the base oil (SN1) blended with the additives in different mass fractions (reproduced with permission from ref. [24], Copyright 2015 American Chemical Society).

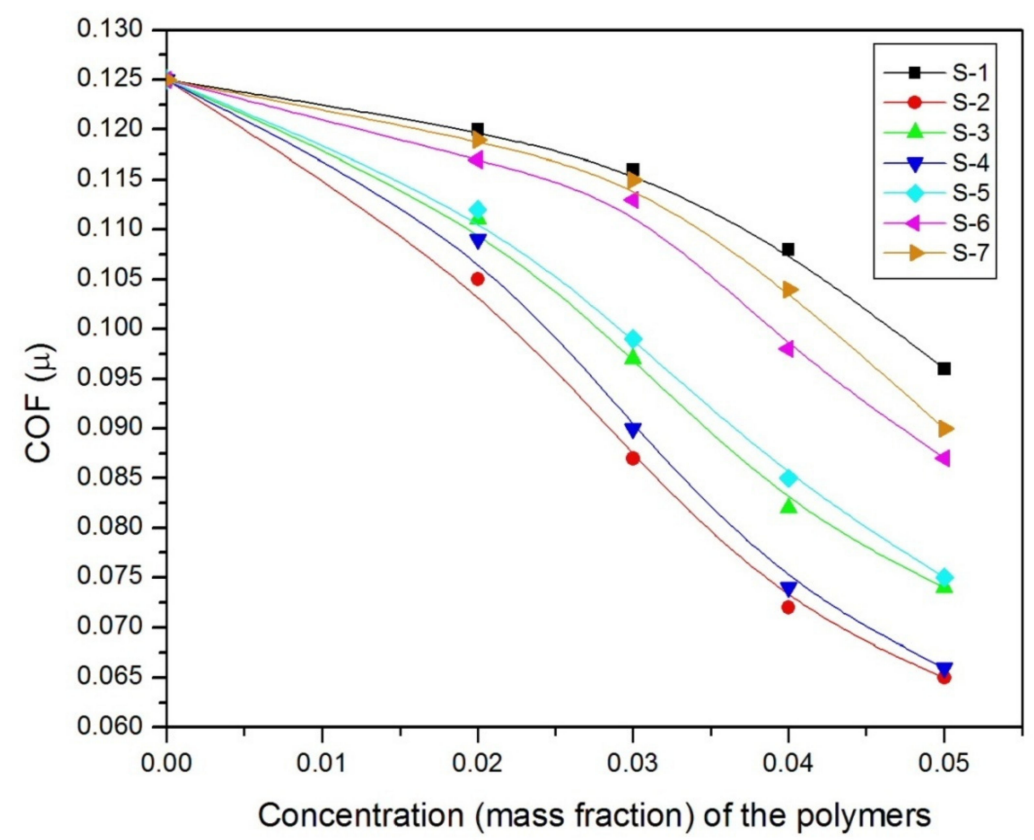

Figure 3. Coefficient of friction (COF) values of the SN1 base fluid blended with additives in different mass fractions (reproduced with permission from ref. [24], Copyright 2015 American Chemical Society). 
In another study, a homopolymer of SBO and its copolymers with MA and methyl methacrylate (MMA) as a multifunctional additive for lubricating oil was synthesized by the atom transfer radical polymerization (ATRP) method [22]. Polymerization was carried out at $90{ }^{\circ} \mathrm{C}$ with microwave irradiation (MI) using anhydrous $\mathrm{FeCl}_{3}$ as a catalyst, diethylenetriamine (DETA) as a ligand, AIBN as the initiator, toluene as a solvent, and metallic Fe as the reducing agent. The characterization of the prepared polymers was performed by spectral (NMR, IR) and gel permeation chromatography (GPC) analysis. The evaluations of the polymers regarding their antiwear (AW) pour point depressant (PPD), and viscosity modifier/viscosity index improver (VII) performances in different mineral base oils were done by using the standard ASTM methods. The biodegradability test of the polymers was carried out by the soil burial test (SBT) method. The addition of these newly developed biodegradable additives to mineral base stocks enhances the lubricant properties significantly. The control of polymerization through ATRP and the eco-friendly microwave irradiation process produces polymers with excellent PPD, VII, and antiwear performances. Significant enhancement of the VI and PP performances was observed by incorporating acrylates (MA and MMA) in the triglyceride backbone of soybean oil, as depicted in Table 5. The antiwear performance of the lubricants was carried out by applying a weld load of $392 \mathrm{~N} \mathrm{(40} \mathrm{kg)} \mathrm{at} 75^{\circ} \mathrm{C}$ for $60 \mathrm{~min}$. The diameter and rotating speed of the ball were $12.7 \mathrm{~mm}$ and $1200 \mathrm{rpm}$, respectively. Figures 4 and 5 depicted the WSD and $\mathrm{COF}$ values of the formulated lubricants with different percentages of additives in mineral base oil (SN70), respectively.

Table 5. Viscosity index (VI) and pour point values of the base oils blended with the additives at different concentrations [22].

\begin{tabular}{|c|c|c|c|c|c|c|c|c|c|c|c|}
\hline \multirow{2}{*}{ Sample } & \multirow{2}{*}{ Base Oil } & \multicolumn{5}{|c|}{ VI } & \multicolumn{5}{|c|}{ Pour Points $\left(-{ }^{\circ} \mathrm{C}\right)$} \\
\hline & & $0 \%$ & $2 \%$ & $3 \%$ & $4 \%$ & $5 \%$ & $0 \%$ & $2 \%$ & $3 \%$ & $4 \%$ & $5 \%$ \\
\hline \multirow{2}{*}{ S-1 } & SN70 & 80.05 & 102 & 135 & 189 & 207 & -3 & -6 & -7 & -8 & -9 \\
\hline & SN150 & 89.02 & 115 & 142 & 198 & 220 & -6 & -6 & -8 & -9 & -10 \\
\hline \multirow{2}{*}{ S-2 } & SN70 & 80.05 & 120 & 147 & 205 & 221 & -3 & -6 & -9 & -12 & -18 \\
\hline & SN150 & 89.02 & 125 & 161 & 213 & 232 & -6 & -6 & -9 & -15 & -18 \\
\hline \multirow{2}{*}{ S-3 } & SN70 & 80.05 & 128 & 169 & 211 & 229 & -3 & -6 & -8 & -12 & -15 \\
\hline & SN150 & 89.02 & 132 & 174 & 223 & 239 & -6 & -6 & -9 & -12 & -15 \\
\hline \multirow{2}{*}{ S-4 } & SN70 & 80.05 & 136 & 184 & 218 & 232 & -3 & -6 & -6 & -9 & -12 \\
\hline & SN150 & 89.02 & 142 & 201 & 232 & 255 & -6 & -6 & -6 & -10 & -15 \\
\hline \multirow{2}{*}{ S-5 } & SN70 & 80.05 & 140 & 199 & 232 & 240 & -3 & -6 & -7 & -8 & -12 \\
\hline & SN150 & 89.02 & 146 & 212 & 234 & 262 & -6 & -6 & -8 & -9 & -12 \\
\hline
\end{tabular}

S-1: homopolymer of soybean oil (HSBO); S-2: soy oil + 5\% MA (CSBO/MA-5); S-3: Soy oil + 10\% MA (CSBO/MA10); S-4: Soy oil + 5\% MMA (CSBO/MMA-5); S-5: Soy oil + 10\% MMA (CSBO/MMA-10); CSBO: Copolymer of soybean oil. 


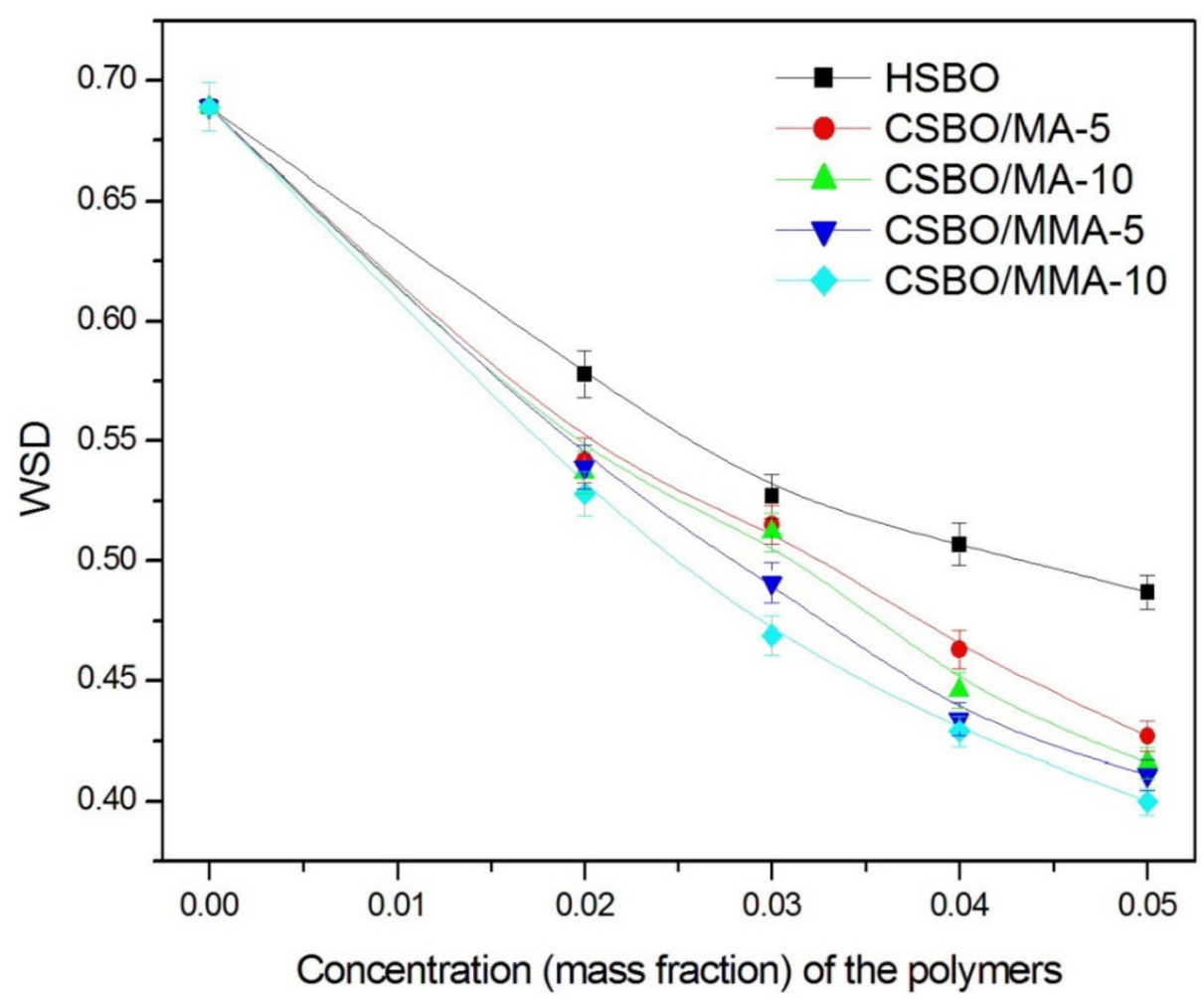

Figure 4. WSD $(\mathrm{mm})$ values of SN70 base oil blended with additives in different mass fractions (reproduced with permission from ref. [22], Copyright 2016 American Chemical Society).

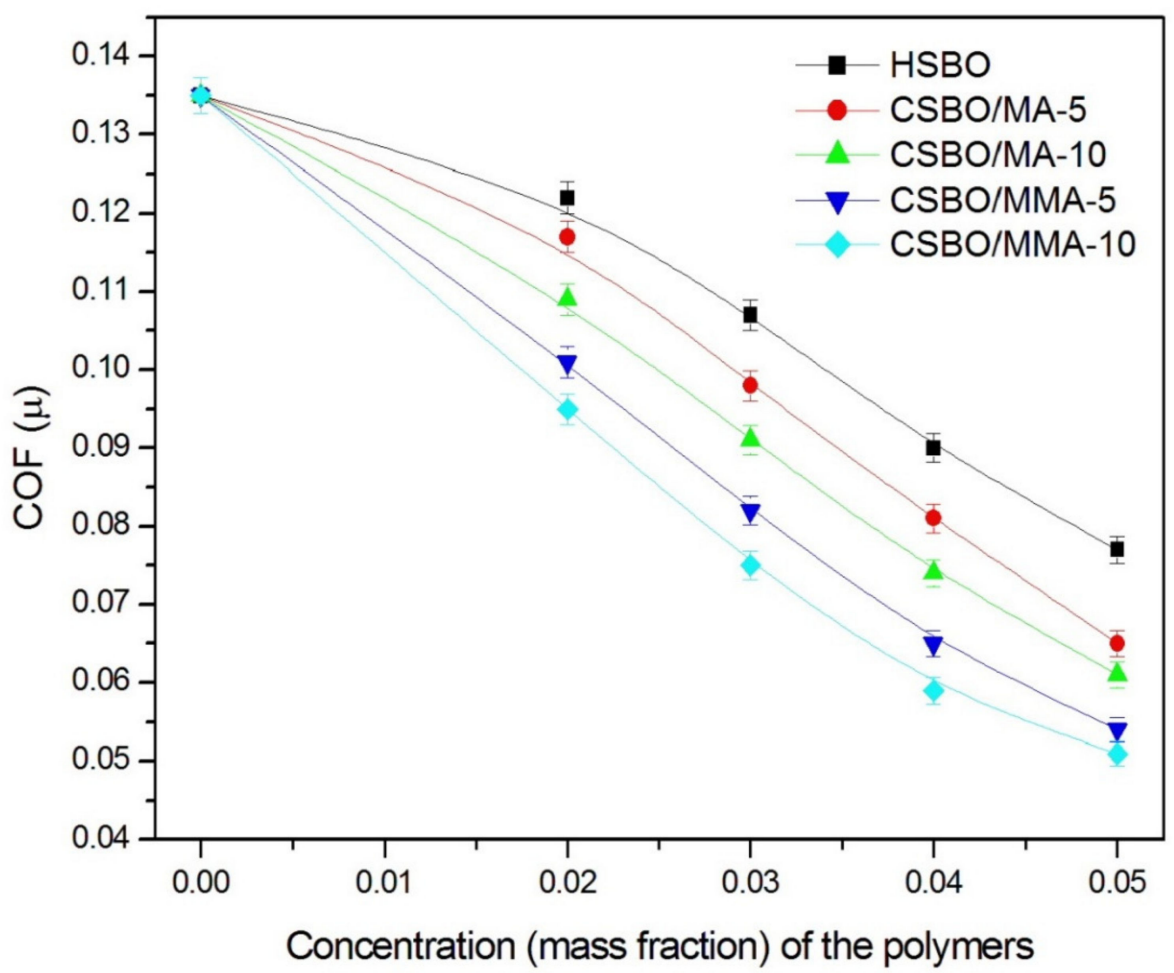

Figure 5. Coefficient of friction of SN70 base oil blended with the additives in different mass fractions (reproduced with permission from ref. [22], Copyright 2016 American Chemical Society).

The tentative structures of the copolymers of SBO with MA and MMA are shown in Figures 6 and 7, respectively. 


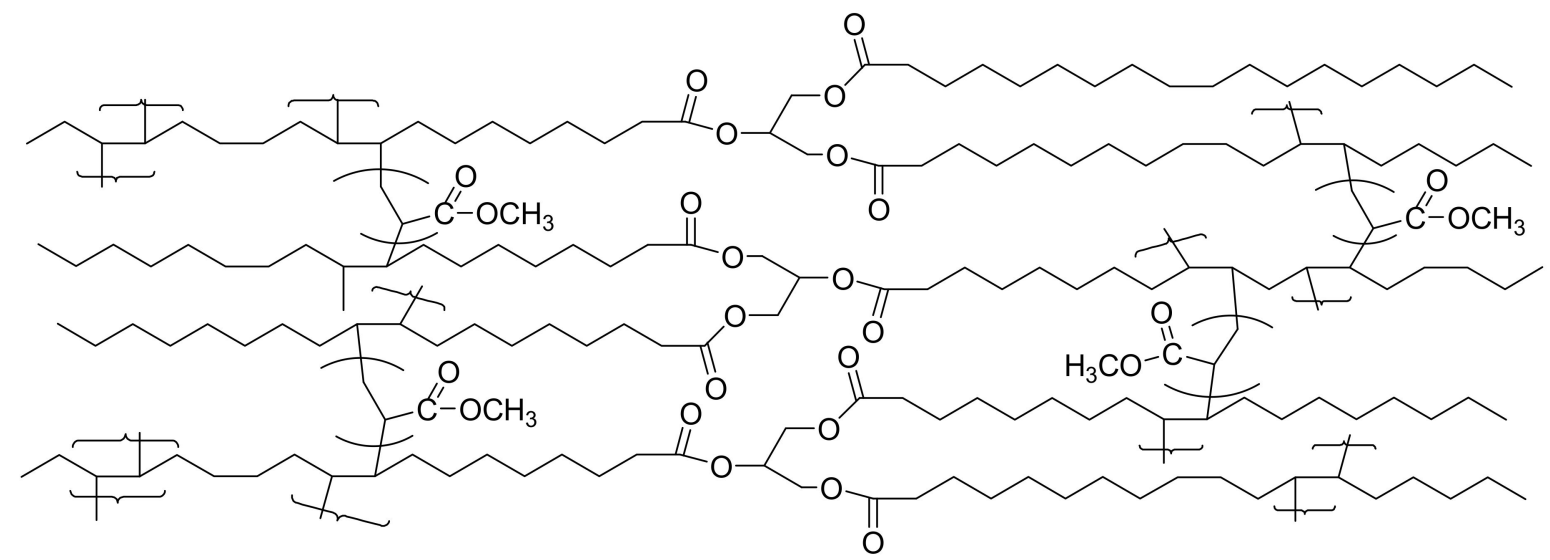

Figure 6. Tentative structure of copolymer of SBO and MA.

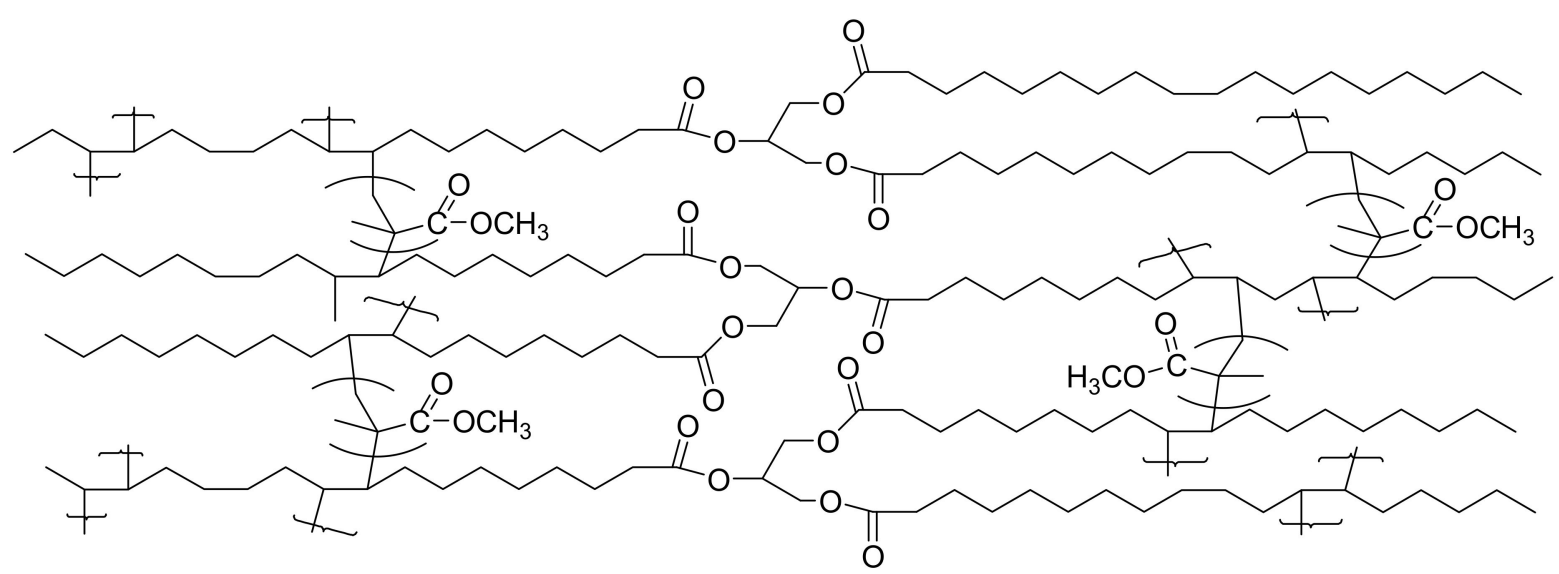

Figure 7. Tentative structure of copolymer of SBO and MMA.

\subsection{Polymers of Sunflower Oil/Derivatives}

The homopolymer of sunflower oil (SFO) and its copolymerization with different mass fractions (5\% and $10 \%$ ) of methyl methacrylate (MMA), decyl acrylate (DA), and styrene have been carried out using the free radical technique [49]. Their characterization and performance evaluation as a pour point depressant in base oils have been investigated. The findings showed that the thermal stability of the sunflower oil-based polymers is enhanced due to the incorporation of monomers (MMA, DA, and styrene) in the backbone of the sunflower oil. The pour points of the copolymer blended base oils are better than those of the homopolymer of sunflower oil (Table 6). 
Table 6. Pour point values of the lubricant compositions [49].

\begin{tabular}{|c|c|c|c|c|c|c|}
\hline \multirow[t]{2}{*}{ Sample } & \multicolumn{2}{|c|}{ Base Oils } & \multicolumn{4}{|c|}{$\begin{array}{c}\text { Pour Points }\left({ }^{\circ} \mathrm{C}\right) \text { of Lubricants with Additives a } \\
\text { Different Percentages }\end{array}$} \\
\hline & & & $0 \%$ & $2.5 \%$ & $5 \%$ & $10 \%$ \\
\hline \multirow{4}{*}{ P-1 } & \multirow{2}{*}{ BO1 } & $\mathrm{S} 1$ & -3 & -6 & -6 & -6 \\
\hline & & S2 & -3 & -3 & -3 & -3 \\
\hline & \multirow{2}{*}{$\mathrm{BO} 2$} & $\mathrm{~S} 1$ & -6 & -9 & -9 & -9 \\
\hline & & S2 & -6 & -9 & -9 & -9 \\
\hline \multirow{4}{*}{ P-2 } & \multirow{2}{*}{ BO1 } & $\mathrm{S} 1$ & -3 & -12 & -12 & -12 \\
\hline & & $\mathrm{S} 2$ & -3 & -12 & -15 & -15 \\
\hline & \multirow{2}{*}{$\mathrm{BO} 2$} & $\mathrm{~S} 1$ & -6 & -15 & -15 & -15 \\
\hline & & $\mathrm{S} 2$ & -6 & -15 & -15 & -15 \\
\hline \multirow{4}{*}{ P-3 } & \multirow{2}{*}{ BO1 } & $\mathrm{S} 1$ & -3 & -12 & -12 & -12 \\
\hline & & S2 & -3 & -15 & -15 & -12 \\
\hline & \multirow{2}{*}{$\mathrm{BO} 2$} & $\mathrm{~S} 1$ & -6 & -15 & -12 & -12 \\
\hline & & $\mathrm{S} 2$ & -6 & -15 & -12 & -12 \\
\hline \multirow{4}{*}{ P-4 } & \multirow{2}{*}{ BO1 } & S1 & -3 & -15 & -12 & -12 \\
\hline & & S2 & -3 & -15 & -12 & -12 \\
\hline & \multirow{2}{*}{$\mathrm{BO} 2$} & $\mathrm{~S} 1$ & -6 & -15 & -12 & -12 \\
\hline & & $\mathrm{S} 2$ & -6 & -15 & -12 & -12 \\
\hline \multirow{4}{*}{ P-5 } & \multirow{2}{*}{ BO1 } & $\mathrm{S} 1$ & -3 & -15 & -18 & -15 \\
\hline & & S2 & -3 & -15 & -18 & -21 \\
\hline & \multirow{2}{*}{$\mathrm{BO} 2$} & $\mathrm{~S} 1$ & -6 & -18 & -21 & -21 \\
\hline & & S2 & -6 & -18 & -21 & -24 \\
\hline \multirow{4}{*}{ P-6 } & \multirow{2}{*}{ BO1 } & $\mathrm{S} 1$ & -3 & -9 & -9 & -9 \\
\hline & & S2 & -3 & -9 & -9 & -9 \\
\hline & \multirow{2}{*}{$\mathrm{BO} 2$} & $\mathrm{~S} 1$ & -6 & -9 & -12 & -12 \\
\hline & & $\mathrm{S} 2$ & -6 & -9 & -12 & -12 \\
\hline \multirow{4}{*}{ P-7 } & \multirow{2}{*}{ BO1 } & $\mathrm{S} 1$ & -3 & -9 & -9 & -9 \\
\hline & & S2 & -3 & -9 & -9 & -9 \\
\hline & \multirow{2}{*}{$\mathrm{BO} 2$} & $\mathrm{~S} 1$ & -6 & -9 & -9 & -9 \\
\hline & & $\mathrm{S} 2$ & -6 & -9 & -9 & -9 \\
\hline
\end{tabular}

P-1: homopolymer of SFO; P-2: copolymer of SFO and 5\% MMA; P-3: copolymer of SFO and 10\% MMA; P-4 copolymer of SFO and 5\% DA; P-5: copolymer of SFO and 10\% DA; P-6: copolymer of SFO and 5\% styrene; P-7: copolymer of SFO and $10 \%$ styrene.

In another study, homopolymers of SFO prepared by microwave irradiation and thermal methods using BZP as an initiator and without any solvent were used as a multifunctional additive in mineral base oil-based lubricants [28]. The homopolymer prepared by the microwave-assisted method showed better performance particularly as a viscosity index improver, pour point depressant, and antiwear additive, compared to the thermally prepared homopolymer. The viscosity index value and the WSD data of both the SFO polymer are depicted in Figures 8 and 9, respectively. Moreover, the polymers showed significant biodegradability. 


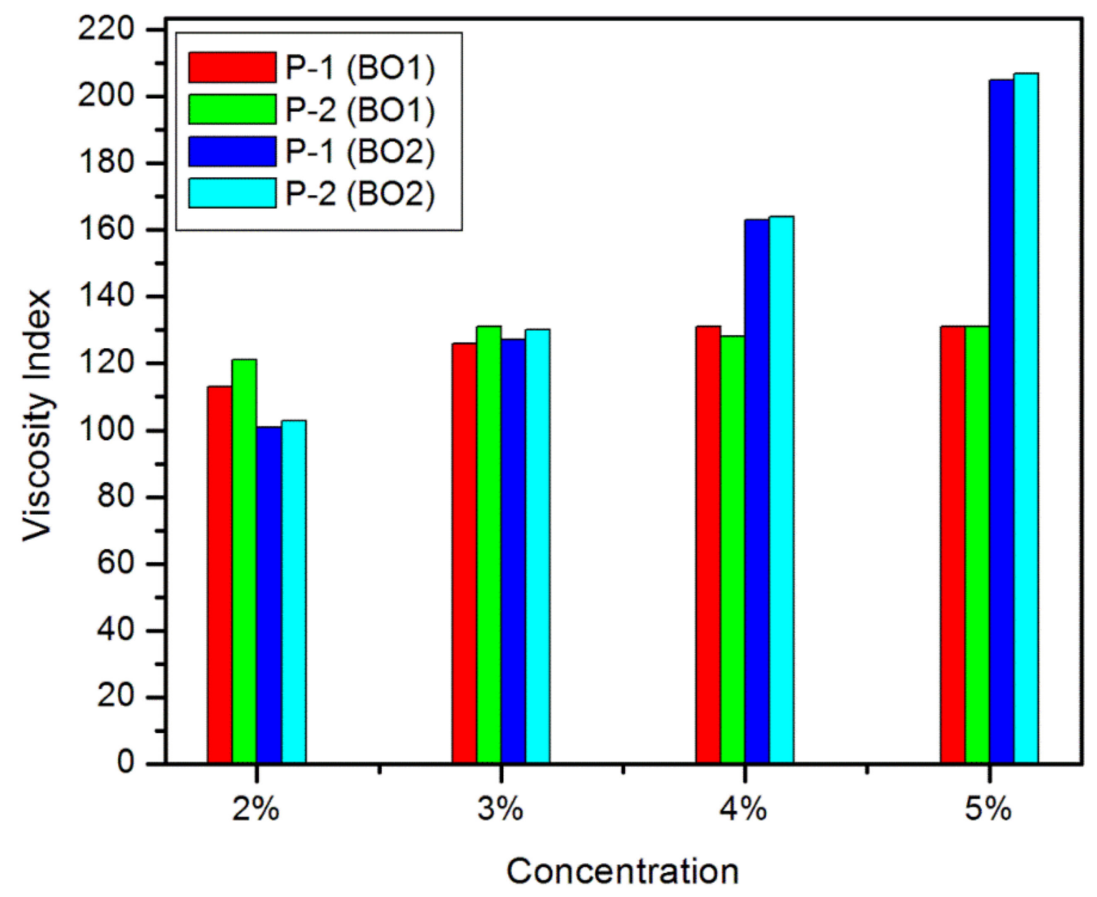

Figure 8. VI values of the two additive doped base oils (BO1 and $\mathrm{BO} 2)$ at different concentrations $(\% w / w)$. [homopolymers SFO in thermal (P-1) and microwave method (P-2)] (reproduced with permission from ref. [28], Copyright 2014 Springer Nature).

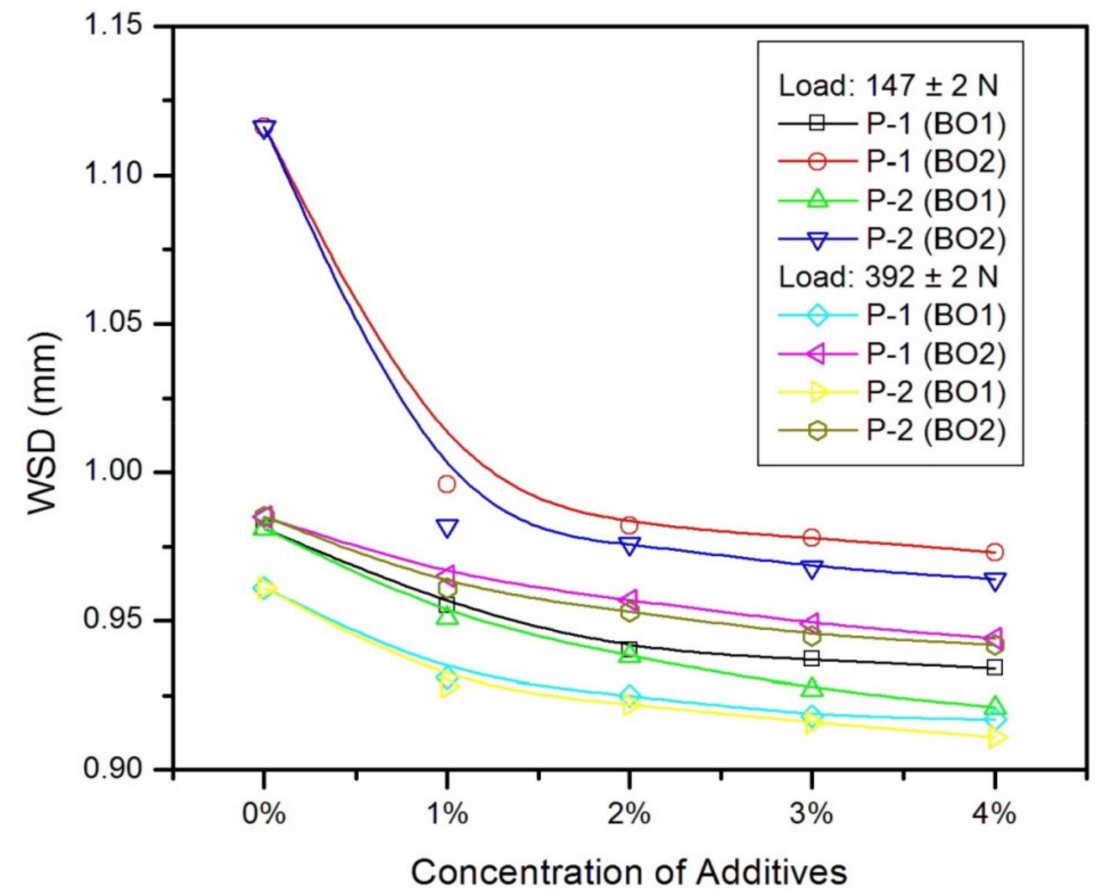

Figure 9. Antiwear performance of the additive doped base oils (BO1 and BO2) evaluated as wear scar diameter (mm) (reproduced with permission from ref. [28], Copyright 2014 Springer Nature).

The copolymers of SFO with methyl methacrylate and decyl acrylate at different percentages were successfully used as a viscosity index improver in three different types of mineral base stocks [50]. In this study, a comparison of the VI performances of the SFO-based polymers with acrylate-based nonbiodegradable polymers was carried out. The results showed that biopolymeric additives have enhanced VI performances in all the base 
stocks compared to acrylate-based polymers in all concentrations. The VI data are given in Table 7.

Table 7. Viscosity index data of lubricants with synthesized additives [50].

\begin{tabular}{|c|c|c|c|c|c|c|}
\hline \multirow{2}{*}{ Sample } & \multirow{2}{*}{ Base Oils } & \multicolumn{5}{|c|}{ VI of Lubricants with Additives at Different Percentages } \\
\hline & & $0 \%$ & $2 \%$ & $3 \%$ & $4 \%$ & $5 \%$ \\
\hline \multirow{3}{*}{ S-1 } & BO1 & 80 & 88 & 81 & 100 & 166 \\
\hline & $\mathrm{BO} 2$ & 89 & 100 & 116 & 139 & 197 \\
\hline & $\mathrm{BO} 3$ & 96 & 96 & 108 & 113 & 148 \\
\hline \multirow{3}{*}{$\mathrm{S}-2$} & BO1 & 80 & 91 & 93 & 93 & 151 \\
\hline & $\mathrm{BO} 2$ & 89 & 108 & 116 & 133 & 197 \\
\hline & BO3 & 96 & 101 & 111 & 113 & 145 \\
\hline \multirow{3}{*}{ S-3 } & BO1 & 80 & 93 & 107 & 121 & 173 \\
\hline & $\mathrm{BO} 2$ & 89 & 89 & 127 & 139 & 212 \\
\hline & BO3 & 96 & 100 & 113 & 122 & 152 \\
\hline \multirow{3}{*}{ S-4 } & BO1 & 80 & 144 & 182 & 203 & 226 \\
\hline & $\mathrm{BO} 2$ & 89 & 95 & 120 & 133 & 210 \\
\hline & BO3 & 96 & 101 & 109 & 112 & 148 \\
\hline \multirow{3}{*}{ S-5 } & BO1 & 80 & 109 & 184 & 230 & 262 \\
\hline & $\mathrm{BO} 2$ & 89 & 97 & 108 & 118 & 215 \\
\hline & BO3 & 96 & 98 & 114 & 121 & 137 \\
\hline
\end{tabular}

S-1: poly(methyl methacrylate); S-2: poly(decyl acrylate); S-3: homopolymer of SFO; S-4: copolymer of SFO with $5 \%$ MMA; S-5: copolymer of SFO with 5\% DA.

SFO-based terpolymers prepared by mixing an alkyl acrylate (octyl/decyl/dodecyl) with SFO and styrene in different ratios through free radical polymerization showed multifunctional performances like viscosity index improver (VII), pour point depressant (PPD), and antiwear when blended in different mineral base oils [29]. The image of the work is given in Scheme 2. In this work, three terpolymers have been prepared by blending octyl acrylate (OA), SFO, and styrene at different weight ratios (1:1:1,3:1:1, and 2:1:1) separately to get three polymers designated by A, B, and C, respectively. Another two polymers consisting of different alkyl acrylates [decyl acrylate (DA) and dodecyl acrylate, (DDA)], SFO, and styrene at the ratio of 2:1:1 $(w / w)$ have also been prepared, which are designated by $\mathrm{D}$ and $\mathrm{E}$, respectively. The investigation disclosed that an effective terpolymeric additive was formed when the ratio of acrylate, sunflower oil, and styrene was 2:1:1. The viscosity index values and pour point values are depicted in Figures 10 and 11, respectively. The antiwear properties in terms of WSD were evaluated by FBWT following the ASTM D 4172-94 method. In this experiment, $392 \mathrm{~N} \mathrm{(40} \mathrm{kg)} \mathrm{load} \mathrm{at} 75^{\circ} \mathrm{C}$ for $60 \mathrm{~min}$ was applied. The WSD data of all the terpolymers are given in Table 8 . The results indicated that when increasing the alkyl chain length, the tribological and VI performances of the prepared terpolymers increased, whereas the PPD performance decreased. 

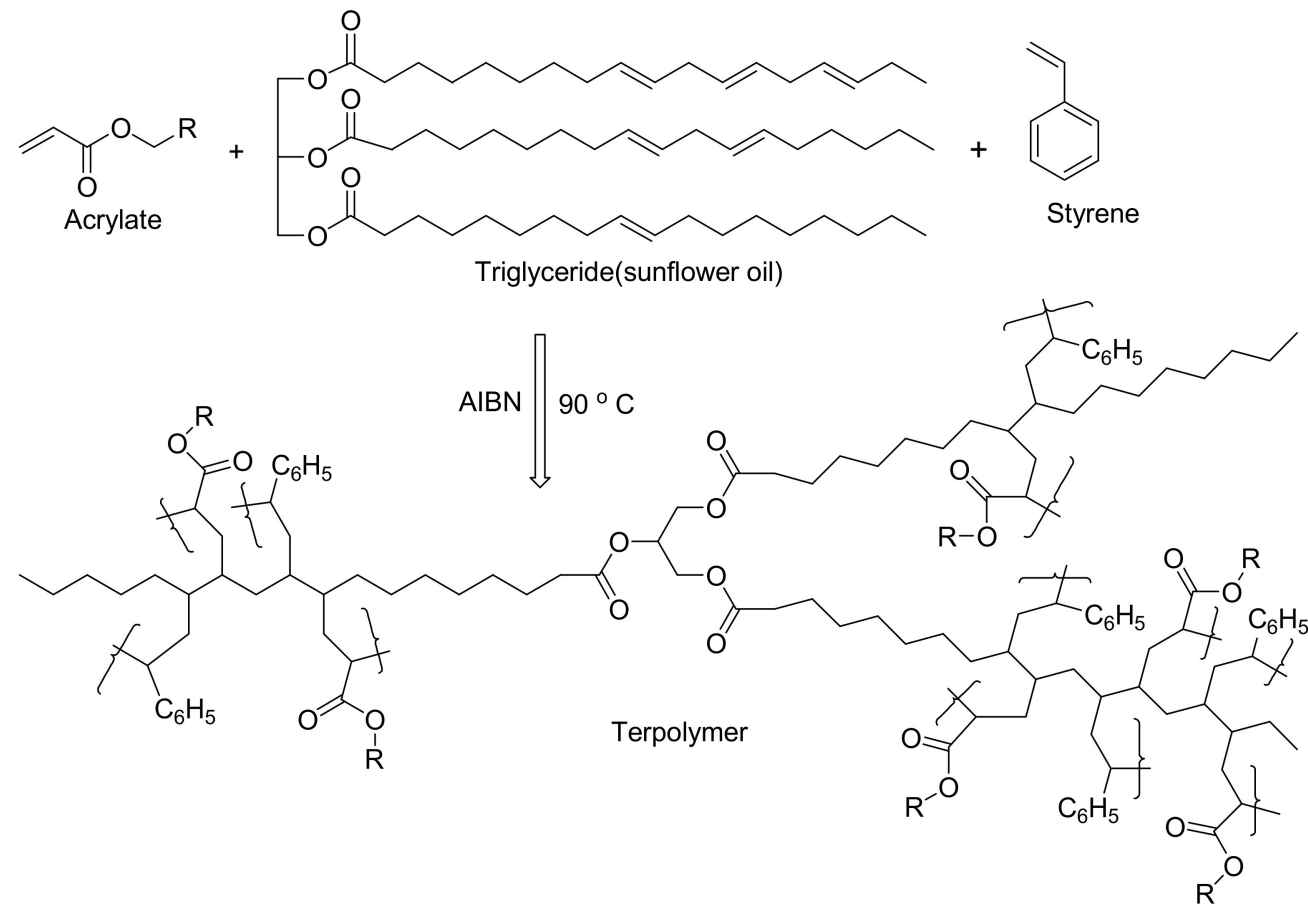

Scheme 2. A probable structure of the terpolymer.

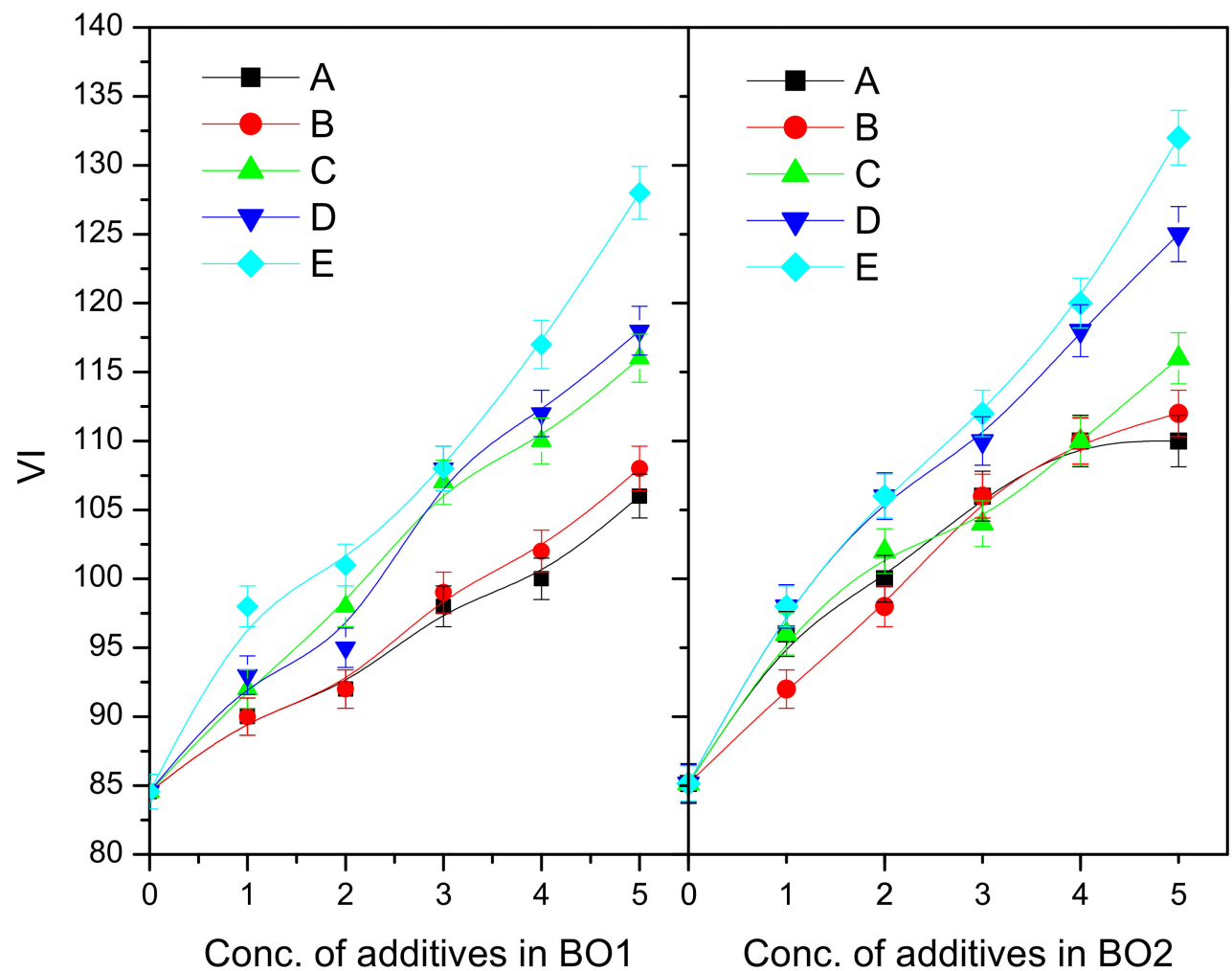

Figure 10. Variation of viscosity index (VI) of the base oils (BO1 and BO2) blended with the polymers at different concentration levels (\% in $w / w)$ (reproduced with permission from ref. [29], Copyright 2016 Springer Nature). 


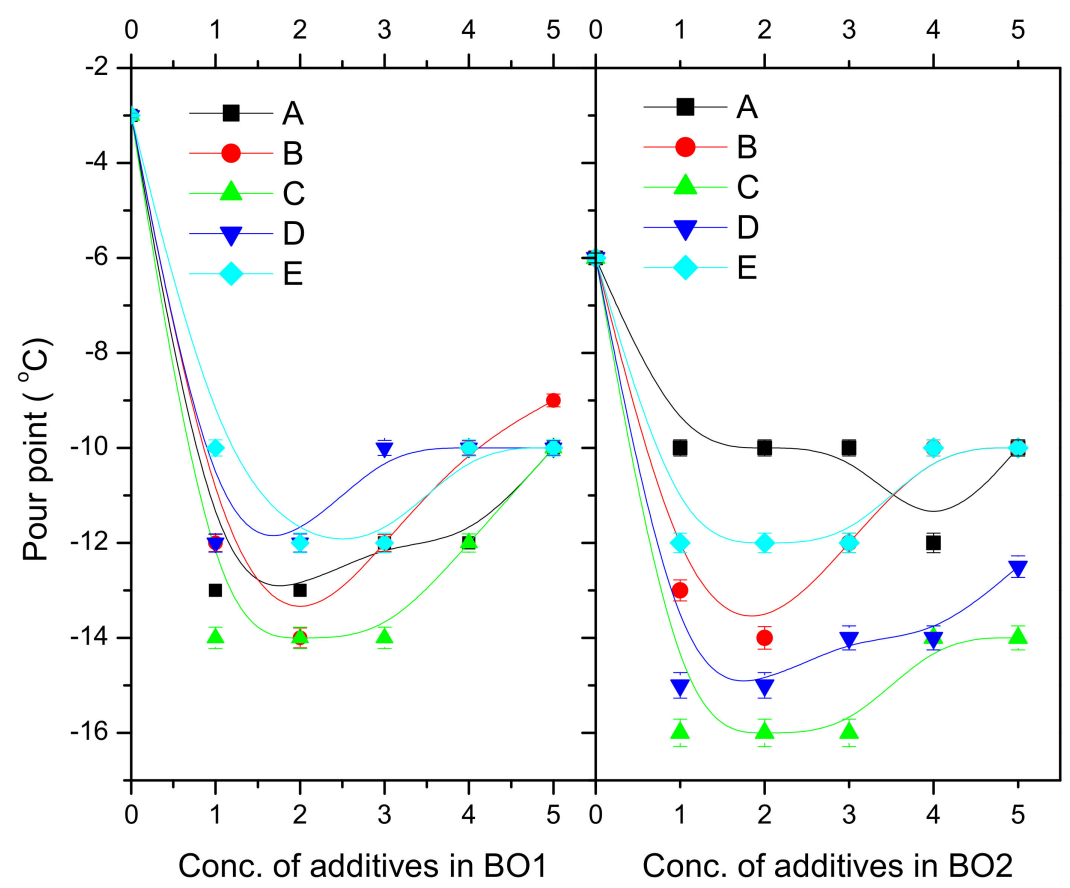

Figure 11. Variation of pour point of the base oils (BO1 and $\mathrm{BO} 2)$ blended with additives at different concentration levels (\% in $w / w$ ) (reproduced with permission from ref. [29], Copyright 2016 Springer Nature).

Table 8. Antiwear property in terms of wear scar diameter (WSD) values (in $\mathrm{mm}$ ) of different lubricant compositions [29].

\begin{tabular}{cccccccc}
\hline \multirow{2}{*}{$\begin{array}{c}\text { Polymer } \\
\text { Code }\end{array}$} & $\begin{array}{c}\text { Base } \\
\text { Fluids }\end{array}$ & \multicolumn{6}{c}{ WSD of Lubricant in $\begin{array}{c}\text { mm at Different Additive Concentrations } \\
(\mathbf{\%} \text { in } \boldsymbol{w} / \boldsymbol{w})\end{array}$} \\
\cline { 3 - 8 } & & $\mathbf{0}$ & $\mathbf{1}$ & $\mathbf{2}$ & $\mathbf{3}$ & $\mathbf{4}$ & $\mathbf{5}$ \\
\hline \multirow{2}{*}{$\mathrm{A}$} & $\mathrm{BO} 1$ & 0.982 & 0.965 & 0.955 & 0.93 & 0.891 & 0.846 \\
& $\mathrm{BO} 2$ & 1.119 & 1.079 & 1.062 & 1.048 & 1.016 & 0.992 \\
$\mathrm{~B}$ & $\mathrm{BO} 1$ & 0.982 & 0.964 & 0.952 & 0.928 & 0.89 & 0.842 \\
& $\mathrm{BO} 2$ & 1.119 & 1.078 & 1.061 & 1.046 & 1.012 & 0.984 \\
$\mathrm{C}$ & $\mathrm{BO} 1$ & 0.982 & 0.951 & 0.944 & 0.919 & 0.875 & 0.833 \\
& $\mathrm{BO} 2$ & 1.119 & 1.072 & 1.053 & 1.038 & 1.006 & 0.974 \\
$\mathrm{D}$ & $\mathrm{BO} 1$ & 0.982 & 0.938 & 0.927 & 0.906 & 0.861 & 0.816 \\
& $\mathrm{BO} 2$ & 1.119 & 1.061 & 1.048 & 1.03 & 0.991 & 0.962 \\
$\mathrm{E}$ & $\mathrm{BO} 1$ & 0.982 & 0.919 & 0.908 & 0.885 & 0.844 & 0.802 \\
& $\mathrm{BO} 2$ & 1.119 & 1.046 & 1.031 & 0.985 & 0.958 & 0.938 \\
\hline
\end{tabular}

A:- O.A + SFO + styrene (1:1:1); B:- O.A + SFO + styrene (3:1:1); C:- O.A + SFO + styrene (2:1:1); D:- D.A + SFO + styrene (2:1:1); D:- D.D.A + SFO + styrene (2:1:1).

\subsection{Polymers of Castor Oil/Derivatives}

Castor oil, which is nonedible and contains a higher percentage of triacylglycerols of ricinoleic acid (85-95\%), can be used as an excellent potential feedstock to prepare a wide variety of materials such as biolubricants (base stock/additive), paints, adhesives, pharmaceuticals, cosmetics, paper, rubber, and agrochemicals [51-54]. The oil's polar functional groups make it efficient to prepare lubricant additives, especially for antiwear and PPD performances. In our earlier studies, the homopolymer of castor oil and its copolymers with dodecyl acrylate (DDA) at different percentage levels $(5,10,15,20$, and $25 \%$ ) were synthesized by a free radical technique, and the additive performances (VII, PPD, antiwear) were evaluated by blending them with mineral base stocks [55]. The viscosity index and pour point values of polymer samples are depicted in Figures 12 and 13. Tribological performance was evaluated by FBWT apparatus applying $392 \mathrm{~N}$ weld load. The 
COF and WSD data of the polymer samples are depicted in Figures 14 and 15, respectively. The biodegradability test of all the polymer samples was carried out through the soil burial test (SBT). The findings showed that the pour points, viscosity index, tribological performances, and biodegradability of the lubricants were enhanced significantly when these additives were blended, and the castor oil-dodecyl acrylate copolymer exhibited better performances compared to the other polymers.

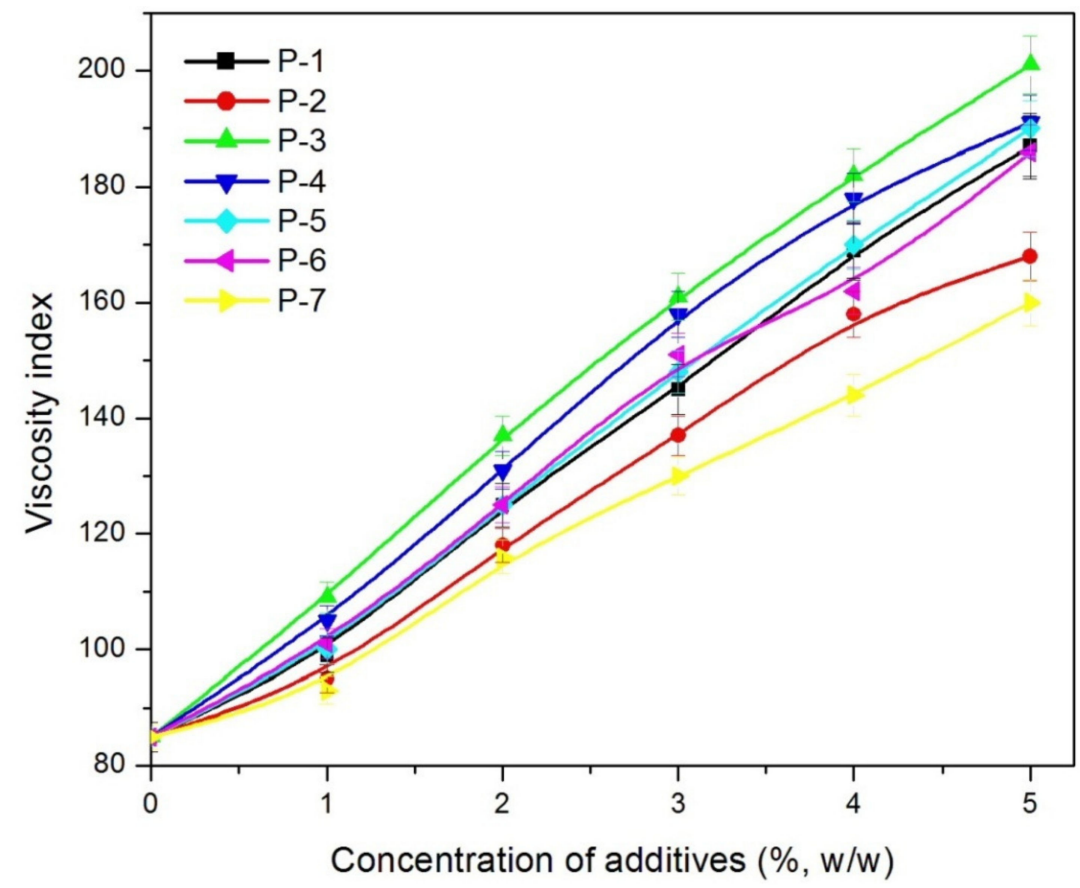

Figure 12. Variation of viscosity index of base oils blended with additives at different concentrations. P-1: Homopolymer of DA; P-2: Copolymer of DA + 5\% CO; P-3: Copolymer of DA + 10\% CO; P-4: Copolymer of DA + 15\% CO; P-5: Copolymer of DA + 20\% CO; P-6: Copolymer of DA + 25\% CO; P-7: Homopolymer of CO (reproduced with permission from ref. [55], Copyright 2016 Springer Nature).

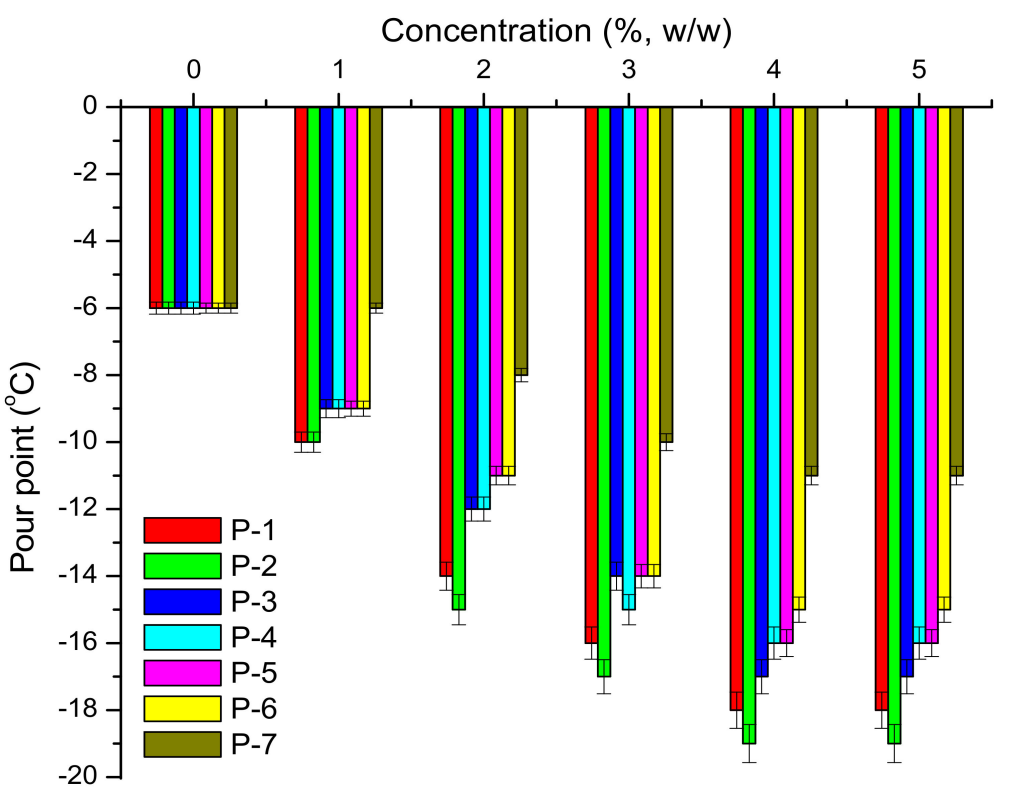

Figure 13. Pour points of the lubricant at different additive concentration levels (reproduced with permission from ref. [55], Copyright 2016 Springer Nature). 


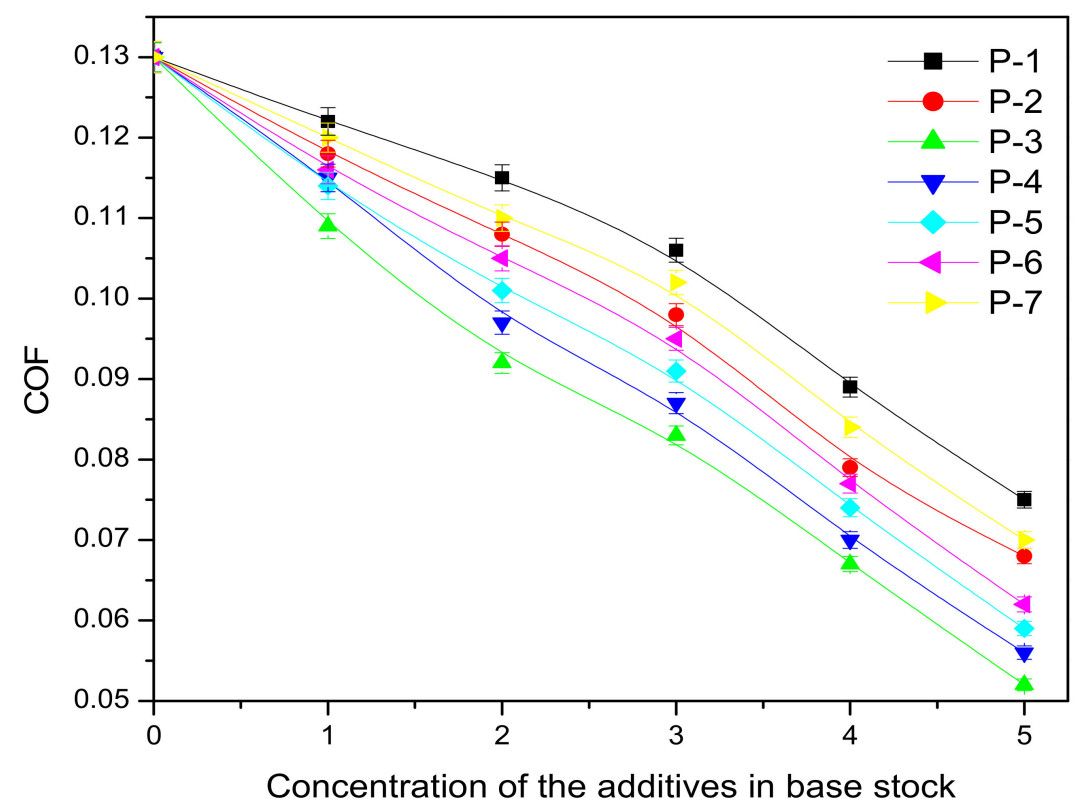

Figure 14. Coefficient of friction (COF) of the lube oil blended with additives at different percentages $(w / w)$ (reproduced with permission from ref. [55], Copyright 2016 Springer Nature).

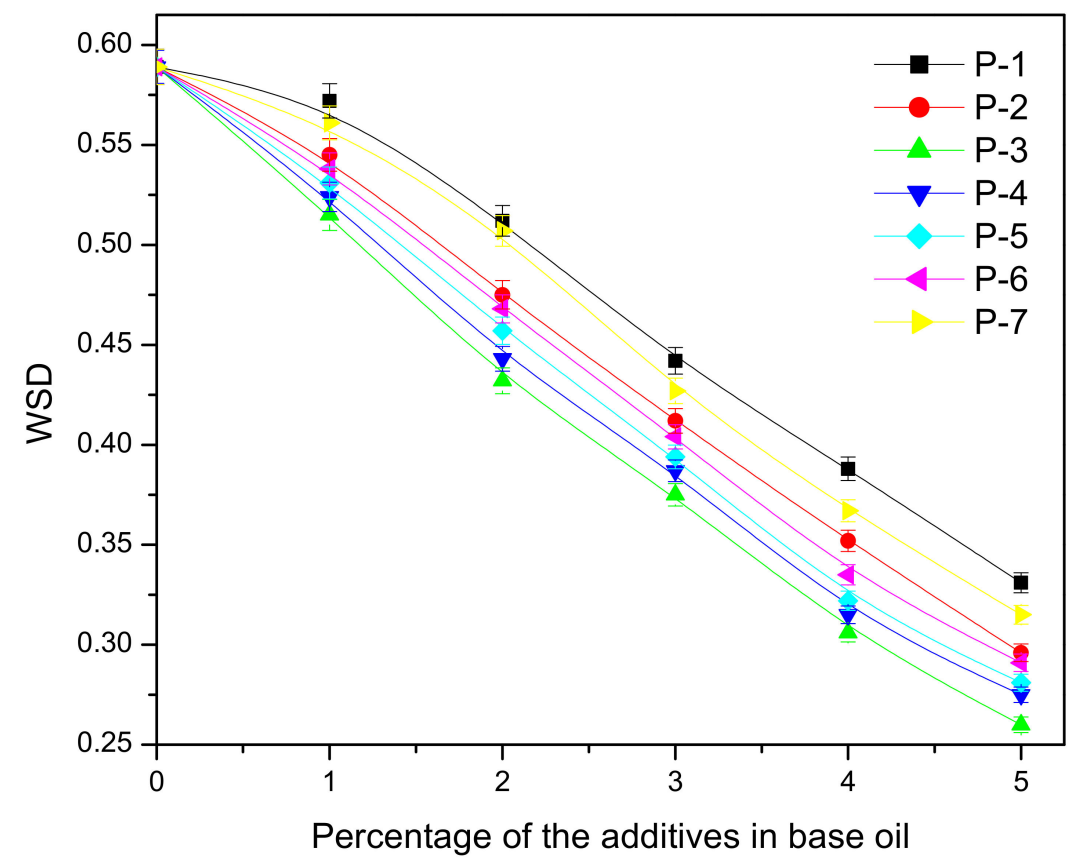

Figure 15. Wear scar diameter (WSD) of the lube oil blended with additives at different percentages $(w / w)$ (reproduced with permission from ref. [55], Copyright 2016 Springer Nature).

In another experiment, the additive performance of the homopolymer of castor oil (CO) and its four copolymers with methyl methacrylate (MMA), dodecyl acrylate (DDA), 1 - decene (1-D), and styrene (ST) with $10 \%(w / w)$ of each have been investigated [27]. The results obtained are mentioned in Table 9. In this study PPD, VII, and tribological performances of the lubricants with these additives at different percentages have been carried out by standard ASTM methods. The SBT method was used to test the biodegradability of the polymers. The copolymers have a better performance compared to homopolymers. The castor-ST copolymer exhibited higher VI, followed by castor-MMA copolymers. The acrylate copolymers are more effective as PPDs and antiwear additives due to their polar ends. 
Table 9. Performance of lubricants at different additive concentrations [27].

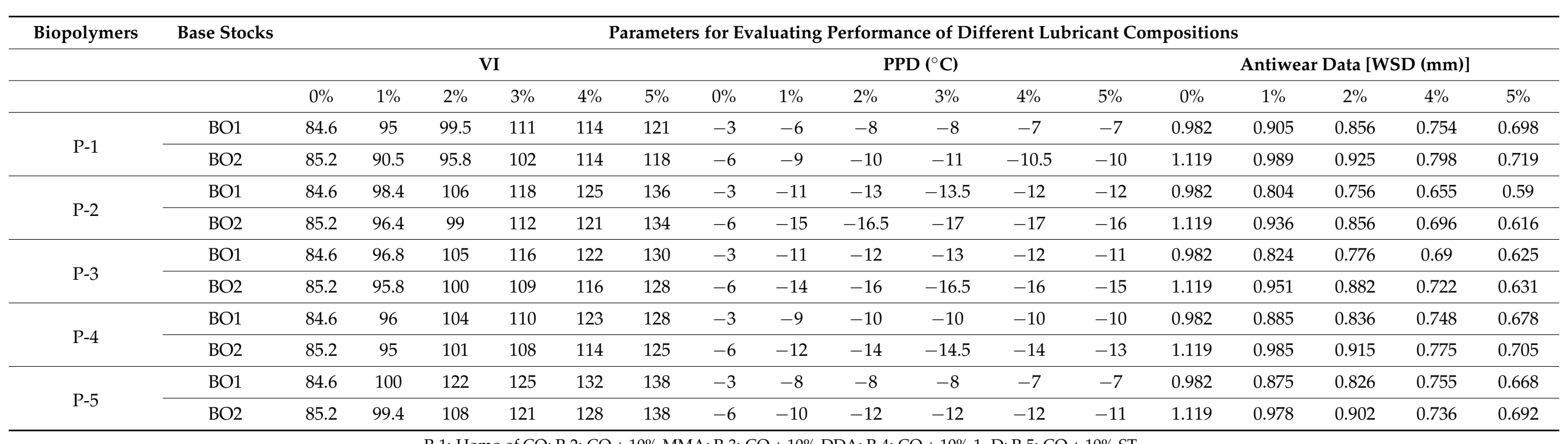

P-1: Homo of CO; P-2: CO + 10\% MMA; P-3: CO + 10\% DDA; P-4: CO + 10\% 1- D; P-5: CO + 10\% ST. 


\subsection{Rice Bran Oil-Based Polymer}

Polymers of rice bran oil (RBO) have also been used as a lubricant additive with effective performances. Copolymers of RBO with 1-decene and decyl acrylate (DA) were prepared and effectively added as a PPD and VII in lubricating oils [56]. The values of the VII and PPD are given in Table 10. In this work, rice bran oil-DA and rice bran oil-1-decene copolymers were prepared by blending different concentrations of DA and 1-decene $(10 \%$, $20 \%$, and $30 \%[w / w])$ in rice bran oil. RBO + DA copolymers are more efficient compared to $\mathrm{RBO}+1$-decene copolymers as a pour point depressant and a viscosity modifier. All the polymers showed significant biodegradability.

Table 10. Viscosity index and pour point data of the lubricants with additives at different concentrations [56].

\begin{tabular}{|c|c|c|c|c|c|c|c|c|}
\hline & \multirow{2}{*}{ Base Oil } & \multirow{2}{*}{ Conc. (\%) } & \multicolumn{6}{|c|}{ Lubricants with Additives } \\
\hline & & & P-1 & P-2 & P-3 & P-4 & P-5 & P-6 \\
\hline \multirow{12}{*}{ VI } & \multirow{6}{*}{ BO1 (85) } & 1 & 112 & 116 & 119 & 100 & 110 & 118 \\
\hline & & 2 & 112 & 118 & 125 & 104 & 112 & 122 \\
\hline & & 3 & 125 & 130 & 132 & 106 & 114 & 128 \\
\hline & & 4 & 125 & 135 & 138 & 118 & 126 & 135 \\
\hline & & 5 & 128 & 136 & 145 & 121 & 128 & 138 \\
\hline & & 6 & 134 & 136 & 146 & 125 & 129 & 137 \\
\hline & \multirow{6}{*}{$\mathrm{BO} 2(80)$} & 1 & 95 & 98 & 101 & 98 & 100 & 104 \\
\hline & & 2 & 97 & 100 & 110 & 109 & 104 & 110 \\
\hline & & 3 & 102 & 115 & 115 & 115 & 118 & 119 \\
\hline & & 4 & 105 & 115 & 123 & 118 & 122 & 125 \\
\hline & & 5 & 115 & 123 & 132 & 131 & 130 & 132 \\
\hline & & 6 & 116 & 128 & 138 & 137 & 129 & 138 \\
\hline \multirow{6}{*}{$\operatorname{PPD}\left({ }^{\circ} \mathrm{C}\right)$} & \multirow{3}{*}{$\begin{array}{l}\text { BO1 } \\
(-3)\end{array}$} & 1 & -9 & -15 & -15 & -9 & -9 & -12 \\
\hline & & 2 & -12 & -18 & -21 & -9 & -12 & -12 \\
\hline & & 3 & -15 & -21 & -24 & -12 & -15 & -15 \\
\hline & \multirow{3}{*}{$\begin{array}{l}\mathrm{BO} 2 \\
(-6)\end{array}$} & 1 & -12 & -15 & -18 & -15 & -21 & -18 \\
\hline & & 2 & -15 & -18 & -21 & -18 & -24 & -24 \\
\hline & & 3 & -15 & -24 & -27 & -21 & -18 & -24 \\
\hline
\end{tabular}

P-1: RBO + DA (10\%); P-2: RBO + DA (20\%); P-3: RBO + DA (30\%); P-4: RBO + 1-decene (10\%); P-5: RBO + 1-decene (20\%); P-6: RBO + 1-decene (30\%).

The biobased polymers synthesized by copolymerization of RBO, peanut oil (PO), and $\beta$ pinene individually with isodecyl acrylate (IDA) in $5 \%$ and $10 \%(w / w)$ ratio performed as VII, PPD, and AW additives in mineral oils (SN1 and SN2) [32]. All the copolymers exhibited better PPD, VII, and tribological performances compared to the homopolymers. The viscosity index and pour point values of all the polymer samples are depicted in Figures 16-19, respectively. The coefficient of friction (COF) data is given in Figure 20. The copolymers of RBO have higher VI values, whereas the copolymers of $\mathrm{PO}$ were found to be most effective as a pour point depressant. In the biodegradability test, the copolymers with higher vegetable oil/ $\beta$-pinene content showed greater degradation in experimental conditions. 


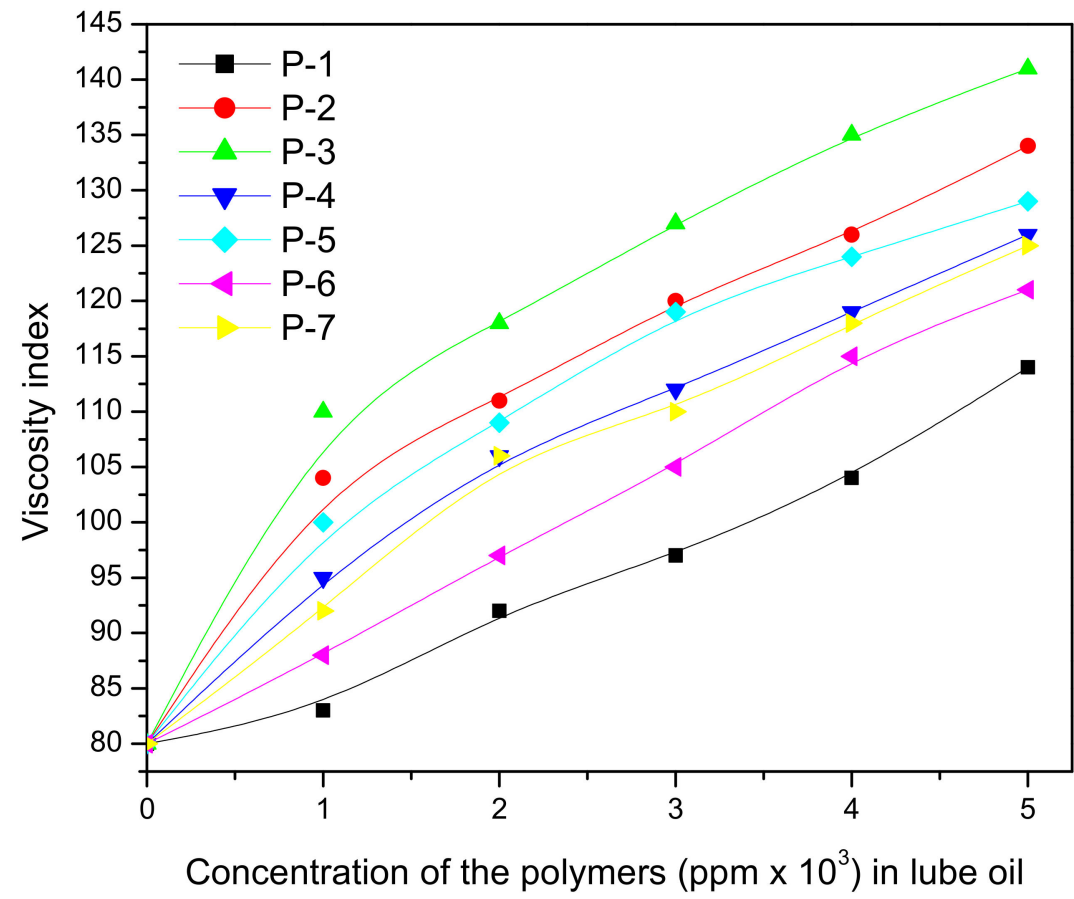

Figure 16. Viscosity index data of SN1 base oil blended with the polymers. P-1: homo polyisodecyl acrylate; P-2: 5\% Rice bran oil + IDA; P-3: 10\% Rice bran oil + IDA; P-4: 5\% Peanut oil + IDA; P-5: 10\% Peanut oil + IDA; P-6: 5\% $\beta$-Pinene + IDA; P-7: 10\% $\beta$-Pinene + IDA.

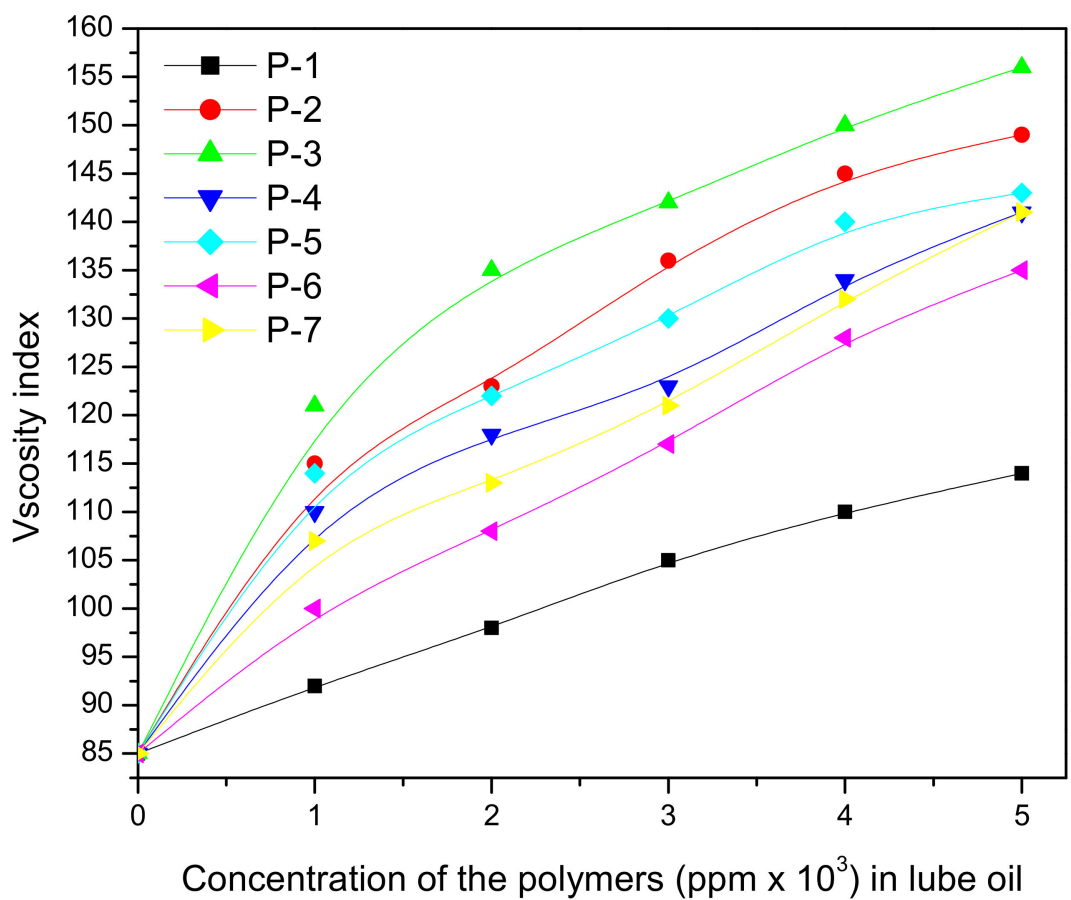

Figure 17. Viscosity index data of SN2 base oil blended with the polymers. 


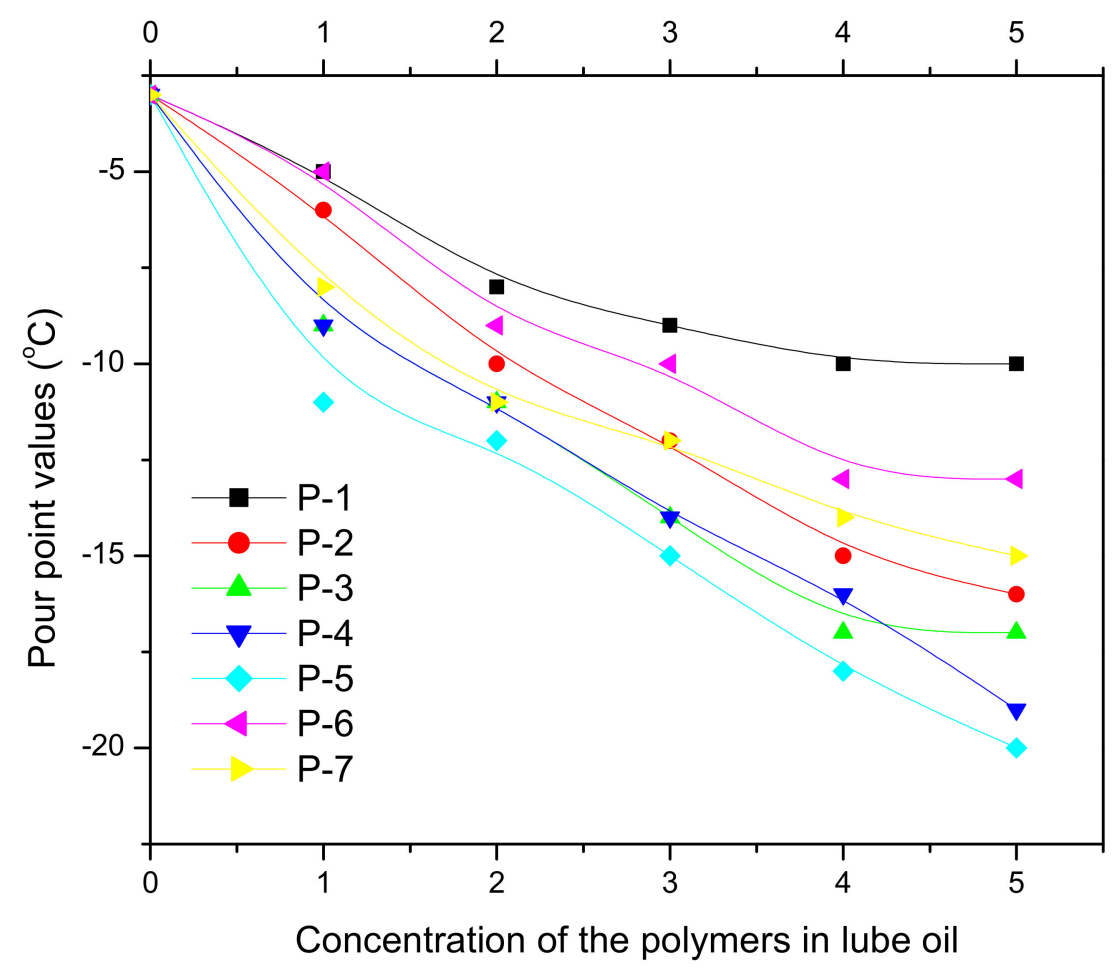

Figure 18. Pour point values of SN1 oil blended with the polymers.

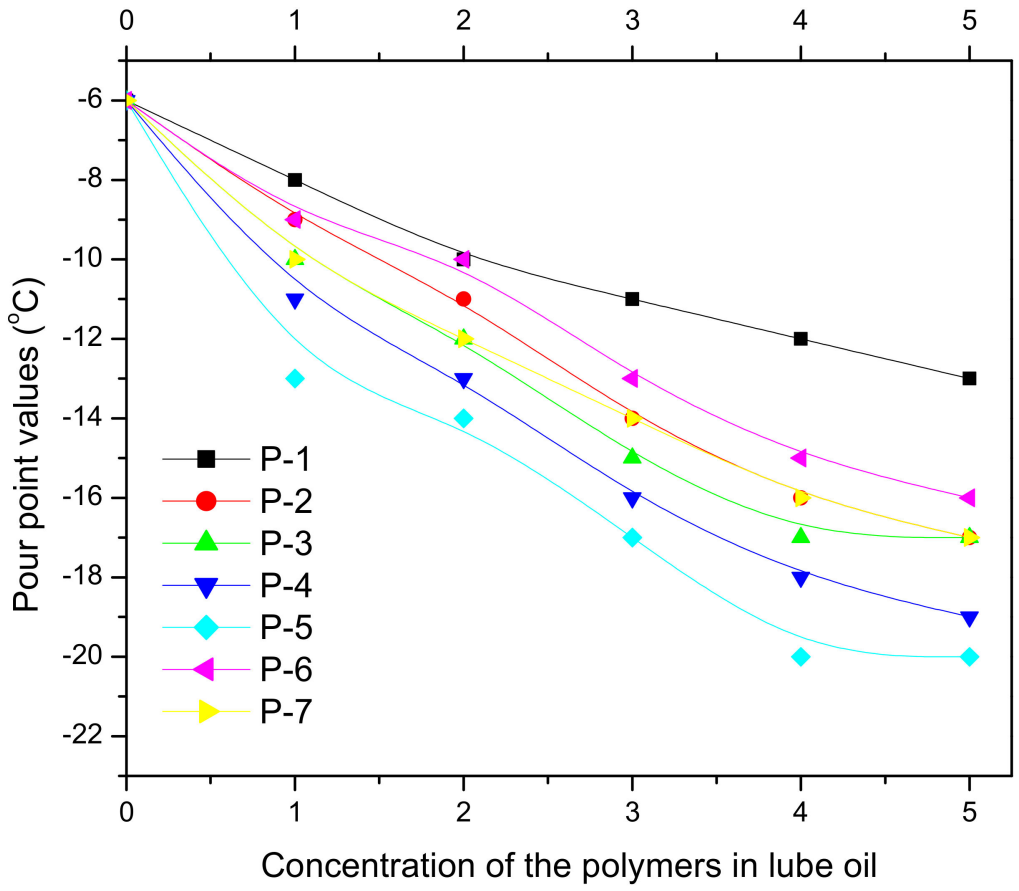

Figure 19. Pour point values of SN2 oil blended with the polymers. 


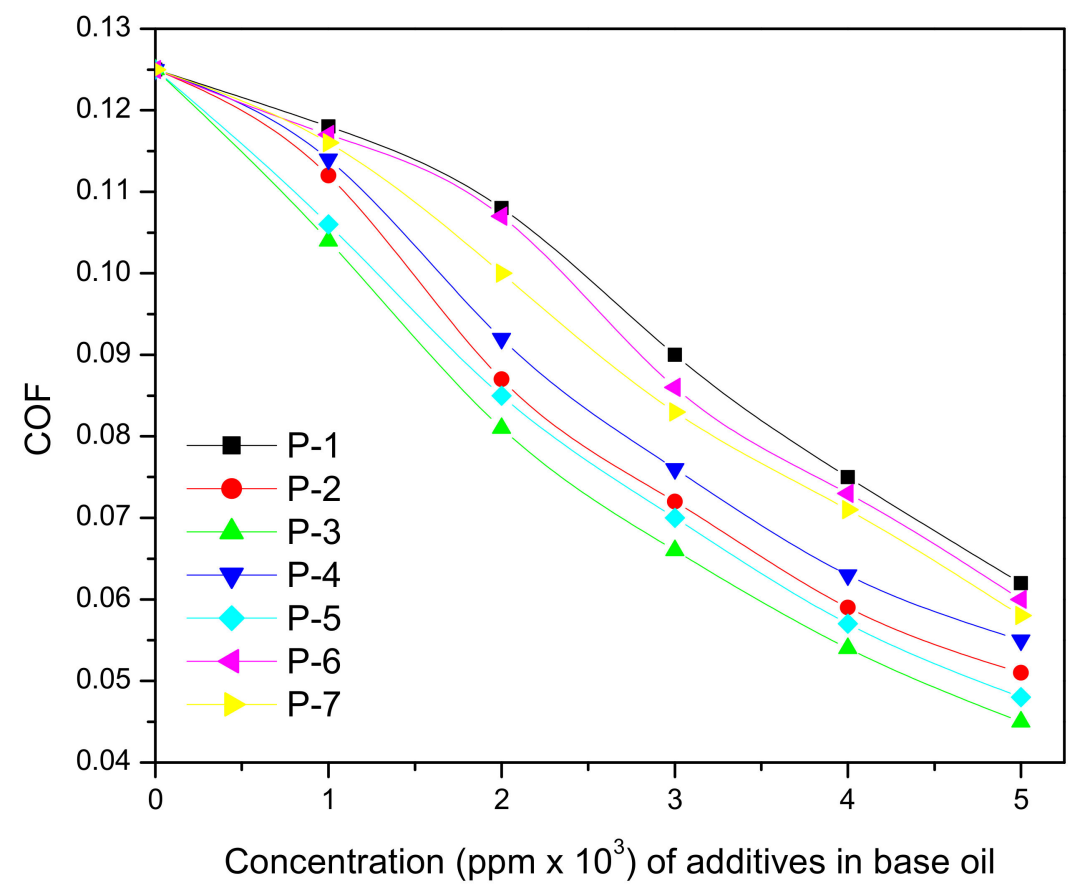

Figure 20. Coefficient of friction (COF) of SN1 base oil blended with the polymeric additives at different concentration levels applying $392 \mathrm{~N}$ weld load (reproduced from ref. [32], copyright 2017 John Wiley and Sons).

\subsection{Olive Oil-Based Polymers}

Olive oil is another potential candidate for preparing bioadditives. Being cheaper and having a higher percentage (about $86 \%$ ) of active unsaturation, the homopolymers and copolymers of olive oil have been found to have a positive impact on lubricant performances. Copolymers of isodecyl acrylate (IDA) with olive oil, prepared by free radical pathways, were investigated as a pour point depressant (PPD) and a viscosity modifier/viscosity index improver (VII) in different base oils (mineral) by standard ASTM methods [33]. In this study, four different copolymers of olive oil and IDA were prepared with varying olive oil concentrations in the mass $(2.5,5,7.5$, and $10 \%)$. The biodegradability test (SBT method) and thermal stability (TGA analysis) of the copolymers have also been investigated. The copolymer with increased olive oil content was found to be thermally more stable and showed the best performance as PPD and VII among all the copolymers. Saha et al. evaluated the performances of lubricants additized with copolymers of olive oil and dodecyl methacrylate (DDMA) [57]. They synthesized four different copolymers of DDMA and olive oil with varying olive oil concentrations in the mass $(2 \%, 4 \%, 6 \%$, and $8 \%$ ) in a focused monomode microwave oven using BZP as radical initiator. The properties, mainly viscosity indices and pour points of the lubricant compositions, have been extensively analyzed by ASTM methods. The copolymers with a higher content of olive oil indicated a higher VI and lower pour points.

\subsection{Almond Oil-Based Polymers}

In another study, the additive performances of almond oil-based polymers [58] have been disclosed. The homopolymer of almond oil and its copolymers with decyl acrylate in two different concentrations (5 and 10\%, $(w / w)$ ) were prepared, and their additive performances were evaluated.

The additive performances were improved due to the incorporation of an acrylate monomer in the triglyceride backbone of almond oil. 


\subsection{Palm Oil-Based Polymers}

The homopolymer of palm oil and its copolymers with decyl acrylate at different concentrations were evaluated as PPD and VII in mineral lubricants by standard ASTM methods. All the polymers showed excellent additive performances with significant biodegradability [34]. Liew et al. disclosed the application of palm oil methyl ester (POME) as a friction-reducing additive for mineral base oil [59]. Table 11 showed the wear scar diameter data of the base oil blended with POME at two different concentrations. It was further confirmed from the data that bioadditives increase the tribological performance of lubricants.

Table 11. Antiwear properties of mineral oil blended with POME additive at two different weld loads [59].

\begin{tabular}{ccccc}
\hline \multirow{2}{*}{ Lubrication Condition } & Weld Load & \multicolumn{3}{c}{ Average Wear Scar Diameter } \\
\cline { 3 - 5 } & $\mathbf{( N )}$ & $\mathbf{3 0 0 ~ N}$ & $\mathbf{6 0 0 ~ N}$ & $\mathbf{8 0 0 ~ N}$ \\
\hline Mineral oil (without palm oil methyl ester) & 1200 & 0.28 & 1.97 & 2.6 \\
\hline Mineral oil (with 5 vol\% palm oil methyl ester) & 1450 & 0.29 & 1.79 & 2.3 \\
\hline
\end{tabular}

\subsection{Jojoba Oil-Based Polymers}

The research team guided by Nasser (2015) prepared a homopolymer of jojoba oil along with its six copolymers with monomers of different alkylacrylates (dodecyl-acrylate, tetradecyacrylate, and hexadecyacrylate) and different $\alpha$-olefins (1-dodecene, 1-tetradecene, and 1-hexadecene) separately with a 1:2 molar ratio [38]. The performances of these polymers as a VII and a PPD were evaluated after their characterizations. It has been found that the viscosity index increases with an increase in the alkyl chain length of both $\alpha$-olefins, and acrylate monomers, while the pour point improved for additives based on alkyl acrylate.

\subsection{Preparation of Vegetable Oil-Based Polymer Nanocomposites and Their Application as an Antifriction Additive for Biolubricants}

The use of polymer nanocomposites (PNC) as an antiwear additive for lubricants has become very popular recently. With increasing environmental concerns, the use of bioresources to prepare polymer biocomposites has come into focus [60,61]. The polymerization of vegetable oils/derivatives in the presence of different organic or inorganic nanoparticles produces vegetable oil polymer- nanocomposites, which are widely applied in the automotive industry for tribological applications [62]. Bhuyana et al. [63] have disclosed the tribological properties of novel biobased nanocomposites prepared by the cationic copolymerization of conjugated low-saturated soybean oil with styrene and divinylbenzene in the presence of reactive organomodified montmorillonite (VMMT) clay of different concentrations $(0 \%, 1 \%$, and $5 \% w / w)$ as the reinforcing phase and modified boron trifluoride diethyl etherate (BFE) as the catalyst. The incorporation of nanoparticles in the polymer matrix improved wear-resistant characteristics and thermal stability compared to neat polymers [64]. The coefficient of friction of the composite with $1 \%$ clay concentration is $19.2 \%$ and $22.4 \%$ lower than the ones with $0 \%$ and $5 \%$ clay concentrations, respectively. Thus, the addition of clay by $1 \%(w / w)$ resulted in a nanocomposite material with superior wear behavior compared to the ones with higher and lower clay concentrations. Based on these studies, it can be said that the VMMT in the polymer matrix plays an important role in enhancing the wear behavior of the resulting nanocomposites.

\section{Test of Biodegradability}

A biodegradability test is very important for polymeric materials towards their application in diverse fields in the current global environmental consciousness. This point has to be borne in mind also for the manufacturing of additives for lubricants. Among the various methods to examine the biodegradability of samples [65], the soil burial test method and 
disc diffusion method using different fungal pathogens according to ISO 846:1997 standard have been applied for the above-mentioned lubricants additized with vegetable oil-based polymers [27-29].

\subsection{Disc Diffusion (DD) Method}

In the disc diffusion method, biodegradation was tested against different fungal pathogens, namely Colletotrichum camelliae (CC), Fusarium equiseti (FE), Alternaria alternata (AA), and Colletotrichum gloeosporioides (CG) [49]. In this method, $1.5 \mathrm{~g}$ of each of the polymer samples was incubated in a bacteriological incubator apparatus at $37{ }^{\circ} \mathrm{C}$ for 30 days. The glass apparatus and culture media were autoclaved before use. In preparing the culture media for fungal strains, suitable proportions of potato extract, dextrose, and agar powder were mixed and autoclaved. Fungal growth was indicated by a change in color of the culture media from yellow to black. After 30 days of incubation, polymer samples were recovered from the fungal media and washed with chloroform, purified, and dried in an open vessel. The dried samples were weighed. The biodegradability of polymer samples was also confirmed by studying the FT-IR spectral analysis and molecular weight analysis before and after the disc diffusion test of the polymer samples.

\subsection{The Soil Burial Test (SBT)}

In this method, test samples were prepared by making a film of lubricant samples with soil compost containing microorganisms and then were kept in a bacteriological incubator apparatus at $\mathrm{pH} 7.2,38{ }^{\circ} \mathrm{C}$, moisture $25 \%$, and relative humidity about $60 \%$ for about 90 days. The microorganisms present in the soil help to degrade the lubricant samples. This was performed as per ISO 846:1997 standard [24]. After definite intervals, the sample was recovered, washed with chloroform, purified, and finally dried. The dried samples were weighed. The percentage weight loss (PWL) of the polymeric samples was calculated by the formula:

$$
\mathrm{PWL}=\left[\left(\mathrm{M}_{0}-\mathrm{M}_{1}\right) / \mathrm{M}_{0}\right] \times 100
$$

where $\mathrm{M}_{0}$ is the initial mass and $\mathrm{M}_{1}$ is the remaining mass after SBT and subsequent drying till constant weight.

All the mentioned polymers showed significant biodegradability in both methods. The biodegradable nature of the lubricant samples increased with the increasing percentages of additives. The biodegradability of the homopolymers of vegetable oils showed the highest degradation. The copolymers having a higher percentage of vegetable oils showed more degradation in the tested methods. In the case of soybean oil-based polymers, polymer samples that contain a higher amount of soy oil are more biodegradable. A similar trend was observed for other kinds of vegetable oil-based polymers reported in this review. In the case of sunflower oil, the homopolymer of sunflower oil synthesized by the microwave irradiation method is more biodegradable compared to a thermally prepared one. The copolymer of rice bran oil with decyl acrylate was found to be more biodegradable compared to a rice bran oil-1-decene copolymer. Figure 21 shows the biodegradability test results of soybean oil, castor oil, and rice bran oil-based homopolymers and copolymers, as mentioned above. 


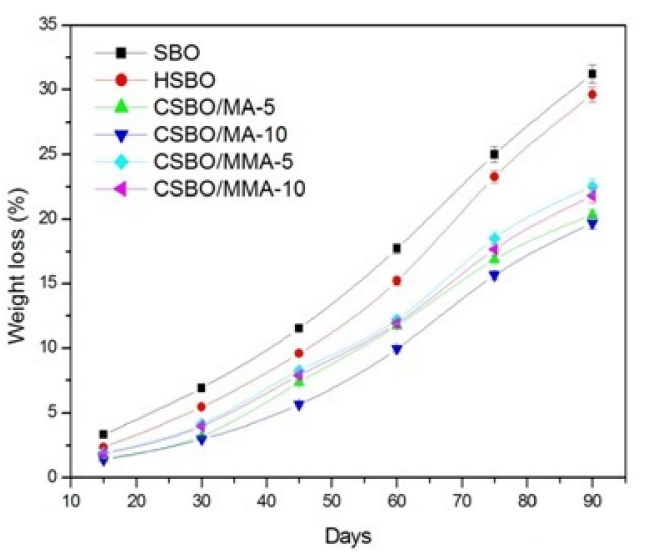

$\mathbf{a}$

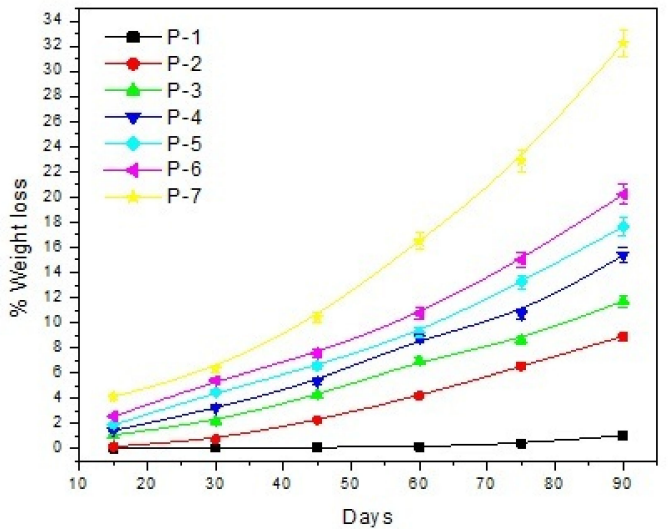

b

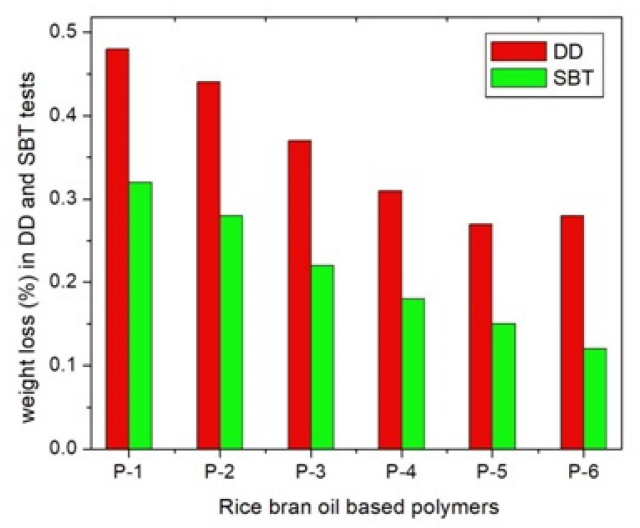

c

Figure 21. Biodegradability test results (SBT and DD); (a) Soybean oil-based polymers [22], (b) Castor oil-based polymers [55], (c) Rice bran oil-based polymers [56].

\section{Conclusions}

In this short review, we highlighted some vegetable oil-based polymeric biomaterials used as lubricating oil additives. The study is significant in the present context of increasing global environmental pollution and decreasing petroleum resources. Generally, vegetable oil has poor thermal stability due to the presence of active unsaturation in the long hydrocarbon chain, which can be removed by homopolymerization or copolymerization with suitable monomers. All these polymeric materials improve the performance of lube oils. Moreover, due to their biodegradability, they are eco-friendly. Copolymers are more efficient as additives compared to homopolymers. The performances of the polymeric additives mainly depend on their morphology, which is directly linked with several factors such as type and ratio of monomers, preparation procedure, and other experimental conditions mentioned above. Copolymers of vegetable oils with alkyl acrylates (methyl acrylate, methyl methacrylate, decyl acrylate, dodecyl acrylate) showed significant enhancement in additive performances, particularly in pour point and antiwear properties compared to others. This is probably due to more polar ends in their macrostructure. On the other hand, copolymers of vegetable oils with styrene, 1-decene have comparatively higher viscosity index values. The viscosity index is directly linked with average molecular weight values which depend on the degree of polymerization of the polymers. A higher degree of polymerization and bigger hydrodynamic volumes of additive molecules in base oils enhanced VI of those copolymers to a greater extent. Another factor found that the microwave-assisted method of homopolymerization of SFO showed better performance compared to the thermal method. Therefore, a microwave-assisted method is more economical and ecofriendly for the synthesis of the homopolymer of SFO. Polymers prepared by ATRP, due to a narrow molecular weight distribution as a result of control on their 
polymerization, are readily soluble in base stocks and therefore more efficient as additives. The excellent additive performances of soybean oil-based homopolymers and copolymers with MA and MMA prepared by the ATRP method proved this. Nonedible castor oil is very attractive as a precursor of polymeric biomaterials because of the presence of its oxy-rich polar functional groups. The copolymer of castor oil with dodecyl acrylate, methyl methacrylate, and styrene exhibited better performances as VI, PPD, and AW additives for lube oil. Castor-styrene copolymers also exhibited a better performance as a VI than castor-methyl methacrylate copolymers. Similarly, other vegetable oils such as rice bran oil, olive oil, palm oil, almond oil-based homopolymers, and copolymers also exhibited additive performances comparable to conventional additives. Transesterified vegetable oil-based additives like palm oil methyl esters also improve tribological properties of mineral base stocks. However, being thermally more stable and of multifunctional character, the vegetable oil-based polymeric additives have some advantages. One challenge in their application is that the solubility of the additives with base stocks is not satisfactory. To improve solubility, sometimes proper diluents are added and the composition is heated to $60^{\circ} \mathrm{C}$ to $70^{\circ} \mathrm{C}$ with vigorous shaking. Another limitation is that the pour points of the lubricants additized with homopolymer of vegetable oils are a little bit lower compared to commercial acrylate-based additives. The pour points of lubricants blended with acrylate copolymers of vegetable oils are comparable with commercial lubricants. However, these bioadditives may be a potential green alternative to commercially available additives in the formulation of the eco-friendly lubricant composition and there is ample scope for research exploring this area.

Author Contributions: Conceptualization, G.K. and B.K.S.; methodology, G.K.; software, K.D.; validation, G.K., K.D., P.G., B.K.S. and S.Z.E.; formal analysis, K.D.; investigation, G.K.; resources, P.G.; data curation, G.K.; writing — original draft preparation, G.K. and K.D.; writing—review and editing, B.K.S.; visualization, S.Z.E.; supervision, P.G., B.K.S., S.Z.E.; project administration, B.K.S.; funding acquisition, B.K.S. All authors have read and agreed to the published version of the manuscript.

Funding: This research received no external funding.

Institutional Review Board Statement: Not applicable.

Informed Consent Statement: Not applicable.

Data Availability Statement: No new data were created or analyzed in this study. Data sharing is not applicable to this article.

Conflicts of Interest: The authors declare no conflict of interest.

\section{References}

1. Miao, S.; Wang, P.; Su, Z.; Zhang, S. Vegetable-oil-based polymers as future polymeric biomaterials. Acta. Biomater. 2014, 10, 1692-1704. [CrossRef]

2. Bähr, M.; Mülhaupt, R. Linseed and soybean oil-based polyurethanes prepared via the non-isocyanate route and catalytic carbon dioxide conversion. Green Chem. 2012, 14, 483-489. [CrossRef]

3. Islam, M.R.; Beg, M.D.H.; Jamari, S.S. Development of Vegetable-Oil-Based Polymers. J. Appl. Polym. Sci. 2014, 131. [CrossRef]

4. Kayode, A.A. Review of vegetable oil-based polymers: Synthesis and applications. Open. J. Polym. Chem. 2015, 5, 34-40. [CrossRef]

5. Florea, M.; Catrinoiu, D.; Paul, L.; Balliu, S. The influence of chemical composition on the pour-point depressant properties of methacrylate copolymers used as additives for lubricating oils. Lubr. Sci. 1999, 12, 31-44. [CrossRef]

6. Mohamed, M.M.; Naga, H.H.A.E.; Meneir, M.F.E. Multifunctional viscosity index improvers. Chem. Technol. Biotechnol. 1994, 60, 283-289. [CrossRef]

7. Liu, W.; Hu, L.; Zhang, Z. Friction and wear of the film formed in the immersion test of oil containing antiwear and extreme pressure additives. Thin Solid Films 1995, 271, 88-91. [CrossRef]

8. Spikes, H.A. Beyond ZDDP. Lubr. Sci. 2008, 20, 77-78. [CrossRef]

9. Maleque, M.A.; Masjuki, H.H.; Sapuan, S.M. Vegetable-based biodegradable lubricating oil additives. Ind. Lubr. Tribol. 2003, 55, 137-143. [CrossRef]

10. Maleque, M.A.; Masjuki, H.H.; Haseeb, A.S.M.A. Effect of mechanical factors on tribological properties of palm oil methyl ester blended lubricant. Wear 2000, 239, 117-125. [CrossRef] 
11. Adhvaryu, A.; Erhan, S.Z.; Perez, J.M. Tribological studies of thermally and chemically modified vegetable oils for use as environmentally friendly lubricants. Wear 2004, 257, 359-367. [CrossRef]

12. Biresaw, G.; Adhvaryu, A.; Erhan, S.Z. Friction properties of vegetable oils. J. Am. Oil. Chem. Soc. 2003, 80, 697. [CrossRef]

13. Mobarak, H.M.; Niza Mohamad, E.; Masjuki, H.H.; Kalam, M.A.; Al Mahmud, K.A.H.; Habibullah, M.; Ashraful, A.M. The prospects of biolubricants as alternatives in automotive applications. Renew. Sustain. Energy. Rev. 2014, 33, 34-43. [CrossRef]

14. Willing, A. Lubricants based on renewable resource-An environmentally compatible alternative to mineral oil products. Chemosphere 2001, 43, 89-98. [CrossRef]

15. Asadauskas, S.; Perez, J.M.; Duda, J.L. Oxidative stability and anti-wear properties of high oleic vegetable oils. Lubr. Eng. 1996, $52,877-882$.

16. Wagner, H. Lubricant base fluids based on renewable raw materials. Their catalytic manufacture and modification. Appl. Catal. A 2001, 221, 429-442. [CrossRef]

17. Zhao, Y.H.; Hupin, S.; Lecamp, L.; Vuluga, D.; Afonso, C.; Burel, F.; Loutelier-Bourhis, C. Thiol-ene chemistry of vegetable oils and their derivatives under UV and air: A model study by using infrared spectroscopy and mass spectrometry. RSC Adv. 2017, 7, 3343-3352. [CrossRef]

18. Salimon, J.; Salih, N.; Yousif, E. Biolubricants: Raw materials, chemical modifications and environmental benefits. Eur. J. Lipid Sci. Technol. 2010, 112, 519-530. [CrossRef]

19. Campanella, A.; Rusto, E.; Baldessari, A.; Baltanás, M.A. Lubricants from chemically modified vegetable oils. Bioresour. Technol. 2010, 101, 245-254. [CrossRef]

20. Karmakar, G.; Ghosh, P.; Sharma, B.K. Chemically modifying vegetable oils to prepare green lubricants. Lubricants $2017,5,44$. [CrossRef]

21. Beran, E.; Łoś, M.; Kmiecik, A. Influence of thermo-oxidative degradation on the biodegradability of lubricant base oils. J. Synth. Lubr. 2008, 25, 75-83. [CrossRef]

22. Karmakar, G.; Ghosh, P. Atom transfer radical polymerization of soybean oil and its evaluation as a biodegradable multifunctional additive in the formulation of eco-friendly lubricant. ACS Sustain. Chem. Eng. 2016, 4, 775-781. [CrossRef]

23. Karmakar, G.; Ghosh, P. Green additives for lubricating oil. ACS Sustain. Chem. Eng. 2013, 1, 1364-1370. [CrossRef]

24. Karmakar, G.; Ghosh, P. Soybean oil as a biocompatible multifunctional additive for lubricating oil. ACS Sustain. Chem. Eng. 2015, 3, 19-25. [CrossRef]

25. Erhan, S.Z.; Sharma, B.K.; Liu, Z.; Adhvaryu, A. Lubricant base stock potential of chemically modified vegetable oils. J. Agric. Food. Chem. 2008, 56, 8919-8925. [CrossRef]

26. Quinchia, L.A.; Delgado, M.A.; Valencia, C.; Franco, J.M.; Gallegos, C. Natural and synthetic antioxidant additives for improving the performance of new biolubricant formulations. J. Agric. Food. Chem. 2011, 59, 12917-12924. [CrossRef]

27. Ghosh, P.; Hoque, M.; Karmakar, G.; Yeasmin, S. Castor oil based multifunctional greener additives for lubricating oil. Curr. Environ. Eng. 2017, 4, 197-206. [CrossRef]

28. Ghosh, P.; Karmakar, G. Evaluation of sunflower oil as a multifunctional lubricating oil additive. Int. J. Ind. Chem. 2014, 5, 1-10. [CrossRef]

29. Ghosh, P.; Hoque, M.; Karmakar, G. Terpolymers based on sunflower oil/alkyl acrylate/styrene as sustainable lubricant additive. Polym. Bull. 2016, 74, 2685-2700. [CrossRef]

30. Xia, Y.; Larock, R.C. Vegetable oil-based polymeric materials: Synthesis, properties, and applications. Green. Chem. 2010, 12, 1893-1909. [CrossRef]

31. Wool, R.P. Polymers and composite resins from plant oils. Bio-Based Polym. Compos. 2005, 56, 113. [CrossRef]

32. Upadhyay, M.; Karmakar, G.; Kapur, G.S.; Ghosh, P. Multifunctional greener additives for lubricating oil. Polym. Eng. Sci. 2017. [CrossRef]

33. Ghosh, P.; Upadhyay, M. Isodecyl acrylate-olive oil copolymers as potential biodegradable additive for lubricating oil. J. Polym. Res. 2016, 23, 100. [CrossRef]

34. Talukdar, S.; Upadhyay, M.; Ghosh, P. Synthesis and performance evaluation of vegetable oil polymer as a multifunctional lube oil additive. Pet. Sci. Technol. 2018, 36, 1-8. [CrossRef]

35. Biresaw, G.; Asadauskas, S.J.; McClure, T.G. Polysulfide and Biobased Extreme Pressure Additive Performance in Vegetable vs Paraffinic Base Oils. Ind. Eng. Chem. Res. 2012, 51, 262-273. [CrossRef]

36. Erhan, S.Z.; Adhvaryu, A.; Sharma, B.K. Poly(hydroxy thioether) Vegetable Oil Derivatives Useful as Lubricant Additives. U.S. Patent 7,279,448 B2, 9 October 2007.

37. Landis, P.S. Telomerized Triglyceride Vegetable Oil for Lubricant Additives. U.S. Patent 5,229,023, 20 July 1993.

38. Nassar, A.M.; Ahmed, N.S.; Nasser, R.M. Jojoba polymers as lubricating oil additives. Pet. Coal 2015, 57, $120-129$.

39. Samad, A.M.; Sinha, S.K. Nanocomposite UHMWPE-CNT Polymer Coatings for Boundary Lubrication on Aluminium Substrates. Tribol. Lett. 2010, 38, 301-311. [CrossRef]

40. Samad, A.M.; Sinha, S.K. Dry sliding and boundary lubrication performance of a UHMWPE/CNTs nanocomposite coating on steel substrates at elevated temperatures. Wear 2011, 270, 395-402. [CrossRef]

41. Lu, Y.; Larock, R.C. Novel polymeric materials from vegetable oils and vinyl monomers: Preparation, properties, and applications. Chem. Sus. Chem. 2009, 2, 136-147. [CrossRef] 
42. Meier, M.A.R.; Metzger, J.O.; Shubert, U.S. Plant Oil Renewable Resources as Green Alternatives in Polymer Science. Chem. Soc. Rev. 2007, 36, 1788-1802. [CrossRef]

43. Tsujimoto, T.; Uyama, H.; Kobayashi, S. Green Nanocomposites from Renewable Resources: Biodegradable Plant Oil-Silica Hybrid Coatings. Macromol. Rapid. Commun. 2003, 24, 711-714. [CrossRef]

44. Fernández-Gutiérrez, M.; Pérez-Köhler, B.; Benito-Martínez, S.; García-Moreno, F.; Gemma Pascual, L.; García-Fernández, M.; Rosa Aguilar, B.; Vázquez-Lasa, J.M.B. Development of Biocomposite Polymeric Systems Loaded with Antibacterial Nanoparticles for the Coating of Polypropylene Biomaterials. Polymers 2020, 12, 1829. [CrossRef]

45. Sánchez-Safont, E.L.; Aldureid, A.; Lagarón, J.M.; Gámez-Pérez, J.; Cabedo, L. Biocomposites of different lignocellulosic wastes for sustainable food packaging applications. Compos. B Eng. 2018, 145, 215-225. [CrossRef]

46. Blayo, A.; Gandini, A.; Le Nest, J.F. Chemical and Rheological Characterizations of Some Vegetable Oils Derivates Commonly Used in Printing Inks. Ind. Crops. Prod. 2001, 14, 155-167. [CrossRef]

47. Hill, K. Fats and Oils as Oleochemical Raw Materials. Pure Appl. Chem. 2000, 72, 1255-1264. [CrossRef]

48. Soutis, C.; Yi, X.; Bachmann, J. How green composite materials could benefit aircraft construction. Sci. China Technol. Sci. 2019, 62, 1478-1480. [CrossRef]

49. Ghosh, P.; Das, T.; Nandi, D.; Karmakar, G.; Mandal, A. Synthesis and Characterization of Biodegradable Polymer-Used as a Pour Point Depressant for Lubricating Oil. Int. J. Polym. Mater. 2010, 59, 1008-1017. [CrossRef]

50. Ghosh, P.; Das, T.; Karmakar, G.; Das, M. Evaluation of acrylate-sunflower oil copolymer as viscosity index improvers for lube oils. J. Chem. Pharm. Res. 2011, 3, 547-556.

51. Ogunniyi, D.S. Castor oil: A vital industrial raw material. Bioresour. Technol. 2006, 97, 1086-1091. [CrossRef] [PubMed]

52. Kunduru, K.R.; Basu, A.; Zada, M.H.; Domb, A.J. Castor oil-based biodegradable polyesters. Biomacromolecules 2015, 16, $2572-2587$. [CrossRef]

53. Mutlu, H.; Meier, M.A.R. Castor oil as a renewable resource for the chemical industry. Eur. J. Lipid. Sci. Technol. 2010, 112, 10-30. [CrossRef]

54. Singh, A.K. Castor oil-based lubricants reduces smoke emission in two stroke engines. Ind. Crop. Prod. 2011, 33, 287-295. [CrossRef]

55. Ghosh, P.; Hoque, M.; Karmakar, G. Castor oil as potential multifunctional additive in the formulation of eco-friendly lubricant. Polym. Bull. 2017. [CrossRef]

56. Ghosh, P.; Dey, K.; Upadhyay, M.; Das, T. Multifunctional biodegradable lube oil additives: Synthesis, characterization, and performance evaluation. Pet. Sci. Technol. 2017, 35, 66-71. [CrossRef]

57. Saha, D.K.; Upadhyay, M.; Ghosh, P. Dodecylmethacrylate-olive oil copolymers as potential biodegradable pour point depressant for lubricating oil. Pet. Sci. Technol. 2017. [CrossRef]

58. Saha, D.K.; Ghosh, P. Almond Oil as Potential Biodegradable Lube Oil Additive: A Green Alternative. J. Polym. Environ. 2017, 26, 2392-2400. [CrossRef]

59. Liew, W.Y.H.; Dayou, S.; Dayou, J.; Siambun, N.J.; Ismail, M.A.B. The effectiveness of palm oil methyl ester as lubricant additive in milling and four-ball tests. Int. J. Surf. Sci. Eng. 2014, 8, 153-172. [CrossRef]

60. Lee, K.Y.; Wong, L.L.C.; Blaker, J.J.; Hodgkinson, J.M.; Bismarck, A. Bio-based macroporous polymer nanocomposites made by mechanical frothing of acrylated epoxidised soybean oil. Green Chem. 2011, 13, 3117-3123. [CrossRef]

61. Miyagawa, H.; Misra, M.; Drzal, L.T.; Mohanty, A.K. Novel biobased nanocomposites from functionalized vegetable oil and organically-modified layered silicate clay. Polymer 2005, 46, 445-453. [CrossRef]

62. Ren, Y.; Zhang, L.; Xie, G. A review on tribology of polymer composite coatings. Friction 2021, 9, 429-470. [CrossRef]

63. Bhuyan, S.; Sundararajan, S.; Lu, Y.; Larock, R.C. A study of the physical and tribological properties of biobased polymer-clay nanocomposites at different clay concentrations. Wear 2010, 268, 797-802. [CrossRef]

64. Bikiaris, D. Can nanoparticles really enhance thermal stability of polymers? Part II: An overview on thermal decomposition of polycondensation polymers. Thermochim. Acta. 2011, 523, 25-45. [CrossRef]

65. Pagga, U.; Beimborn, D.B.; Yamamoto, M. Biodegradability and compostability of polymers-Test methods and criteria for evaluation. J. Environ. Polym. Degr. 1996, 4, 173-178. [CrossRef] 